\title{
Characterisation of walking loads by 3D inertial motion tracking
}

\author{
K. Van Nimmen ${ }^{\mathrm{a}, \mathrm{b}}$, G. Lombaert ${ }^{\mathrm{a}}$, I. Jonkers ${ }^{\mathrm{c}}$, G. De Roeck ${ }^{\mathrm{a}}$, P. Van den Broeck ${ }^{\mathrm{a}, \mathrm{b}}$ \\ ${ }^{a}$ Department of Civil Engineering, KU Leuven, Kasteelpark Arenberg 40, B-3001 Leuven, Belgium \\ ${ }^{b}$ Department of Engineering Technology, KAHO Sint-Lieven, Gebr. De Smetstraat 1, B-9000 Ghent, Belgium \\ ${ }^{c}$ Department of Kinesiology, KU Leuven, Tervuursevest 101, B-3001 Leuven, Belgium
}

\begin{abstract}
The present contribution analyses the walking behaviour of pedestrians in situ by 3D inertial motion tracking. The technique is first tested in laboratory experiments with simultaneous registration of the ground reaction forces. The registered motion of the pedestrian allows for the identification of stride-to-stride variations, which is usually disregarded in the simulation of walking forces. Subsequently, motion tracking is used to register the walking behaviour of (groups of) pedestrians during in situ measurements on a footbridge. The calibrated numerical model of the structure and the information gathered using the motion tracking system enables detailed simulation of the stepby-step pedestrian induced vibrations. Accounting for the in situ identified walking variability of the test-subjects leads to a significantly improved agreement between the measured and the simulated structural response.
\end{abstract}

Keywords: inertial motion tracking, human induced vibrations, footbridge, full-scale testing

\section{Introduction}

Due to their great slenderness, footbridges are very often lively structures, prone to humaninduced vibrations $[1,2]$. Predicting the dynamic response of these structures due to crowd-induced loading has therefore become a critical aspect of the vibration serviceability evaluation $[3,4,5]$. The mathematical load models used in the vibration assessment of these structures are based on traditional direct force measurements utilising an instrumented treadmill or force plates integrated in the walkway [6, 7]. Since human-structure interaction and pedestrian-synchronisation modify the walking behaviour and hence the pedestrian-induced forces [8], they are assumed only to be justifiable in case of structures which do not vibrate perceptibly $[9,10]$.

Recently, visual motion tracking systems have been developed for analysing the human motion and the resulting ground reaction forces. Research in biomedical sciences uses technologically advanced tools, such as the optical marker-based technology of Vicon [11] and CODA [12]. These techniques enable the study of the human motion in laboratory conditions [13, 14], but a remaining challenge is the analysis of the walking behaviour of pedestrians and crowds in situ. To this end, the application of a 3D inertial motion tracking technique developed for the movement science and entertainment industry is examined in the present work.

\footnotetext{
${ }^{*}$ Corresponding author. Tel.: +32 (0)9 2658612 .

Email address: katrien.vannimmen@bwk.kuleuven.be (K. Van Nimmen)
}

Preprint submitted to Journal of Sound and Vibration

Published version: http://dx.doi.org/10.1016/j.jsv.2014.05.022 
(a)

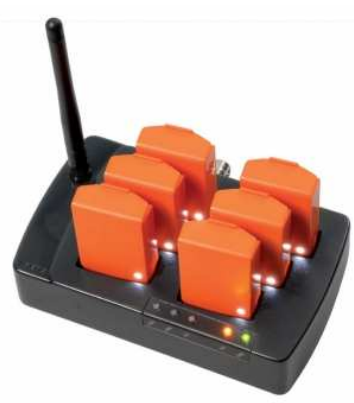

(b)

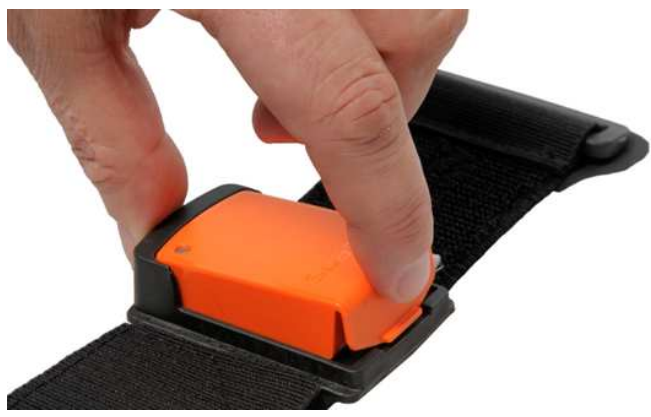

Figure 1: Xsens - MTw Development Kit consisting of multiple wireless inertial units (MTw's) and specially designed click-in full body straps (reproduced with permission of Xsens Technologies B.V. [16]).

The technique is first tested in laboratory experiments involving direct force measurements. An in-depth analysis of the walking behaviour is performed based on the simultaneously registered motion of the test-subject and the resulting ground reaction forces. Secondly, it is verified if the measured ground reaction forces can be accurately simulated using a step-by-step load model available in literature for which the characteristics are determined based on the registered motion of the body segments.

Subsequently, field-tests are performed on a lively lightweight steel footbridge. The structural response and the walking behaviour of the pedestrians are registered simultaneously. The response of the footbridge is simulated based on the numerical model of the structure and information gathered using the motion tracking system. Additional free decay tests are performed to study the influence of standing crowds on the structural dynamic properties.

All procedures were approved by the ethical committee of the university hospital of the KU Leuven and each subject gave written informed consent prior to participation.

The outline of this paper is as follows. First, the 3D motion tracking technique is presented and the experiments in laboratory conditions are examined. Second, the field-tests are presented and the effect of the presence of the pedestrians onto the modal damping ratios is analysed experimentally. In the final section, the results of the numerical simulations are discussed.

\section{Laboratory tests}

The aim of the laboratory experiments is to study the relation between the accelerations of the body segments and the measured ground reaction forces. The experiments are performed in the Movement \& posture Analysis Laboratory Leuven (MALL) at the Department of Kinesiology of KU Leuven [15].

\subsection{Measurement protocol}

The laboratory experiments include both treadmill and overground walking for one subject. The ground reaction forces (GRF's) are registered by an instrumented split-belt treadmill (Forcelink) and three AMTI force plates that are integrated in the walkway (Figure 2). The sampling frequency of both systems is $1 \mathrm{kHz}$.

The main advantage of the treadmill technology is that it allows for the identification of strideto-stride variations [9]. For each walking speed, a measurement time of 2 minutes was considered 
(a)

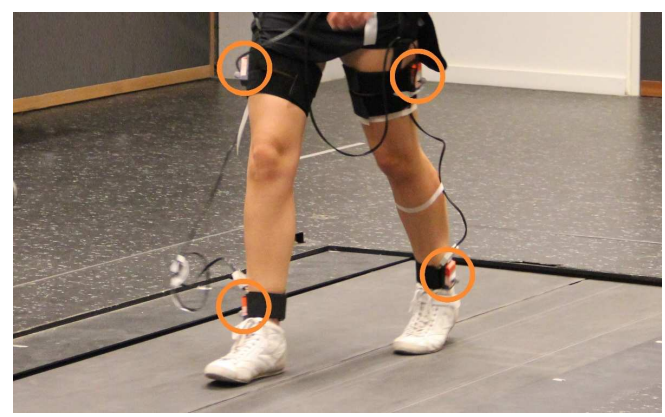

(b)

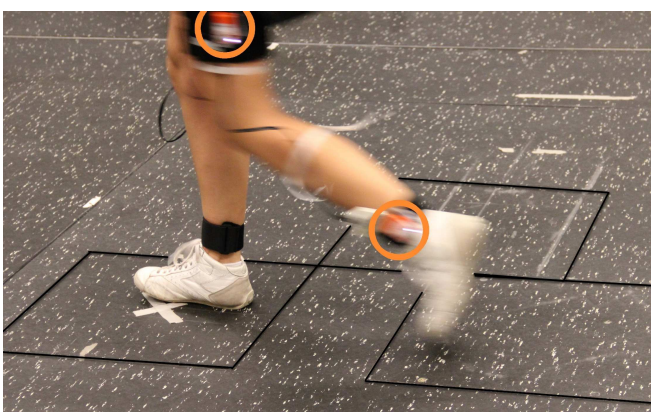

Figure 2: Measurement of ground reaction forces: (a) instrumented split-belt treadmill and (b) three force plates (artificially accentuated) integrated in the walkway.

which allowed for the registration of about 200 steps for each trial. The test-subject (a female in good health, age 27, height $1.65 \mathrm{~m}$ ) is an experienced treadmill user. For each trial, a period of at least 30 seconds was provided to allow the test-subject to adapt his walking behaviour to the selected treadmill speed. In total, 8 walking speeds were considered, varying from 2.5 up to 6.0 $\mathrm{km} / \mathrm{h}$.

In case of overground walking, the different trials considered normal (self-selected), slow and fast walking. The ground reaction forces are in this case registered by three force plates (Figure 2-b) that are embedded in the laboratory floor and covered with identical coverage as the surrounding floor. This configuration allows for the registration of two successive steps for each trial. The subjects are unaware of the plates and take at least 4 steps before and after crossing the plates. Proper foot placement is obtained by modifying the starting position of the subject till foot strike occurs with the whole foot on the force plate. This starting position is then kept constant through the entire session. The subject is instructed to walk back and forth till 12 successful trials are collected.

\subsection{D Inertial Motion Tracking}

The Xsens MTw Development Kit measurement system consists of multiple wireless inertial units (MTw's - figure 1), incorporating 3D accelerometers, gyroscopes, magnetometers (3D compass) and a barometer (pressure sensor). The accompanied Awinda radio protocol ensures time synchronisation between the MTw's across the wireless network. The sensors are securely fixed onto the test-subject with specially designed click-in full body straps (figure 1b). The objective is to identify the main characteristics of the walking behaviour from the motion of the pedestrian tracked by the MTw's, so as to accurately simulate the human induced forces.

The positions of the MTw sensors have been chosen following the recommendations made by the manufacturer to limit the influence of skin motion artifacts $[16,17]$. Using the click-in body straps, the Xsens MTw's are fastened tightly and robustly to the skin. Figure 3 illustrates the configuration setup applied in laboratory conditions. This setup consists of six sensors: two sensors are placed on the outside of each leg, one close to the ankle (sensor label 1 and 2), another on the thigh (sensor label 3 and 4); the fifth sensor is placed as close as possible to the body center of mass $(\mathrm{COM})$ located at the level of the $5^{\text {th }}$ lumbar vertebrae using the pelvis belt and the sixth sensor is placed at the location of the $6^{\text {th }}$ thoracic vertebrae. The sampling frequency of the MTw's was set to $60 \mathrm{~Hz}$, as recommended by the manufacturer for a configuration of 6 sensors. 

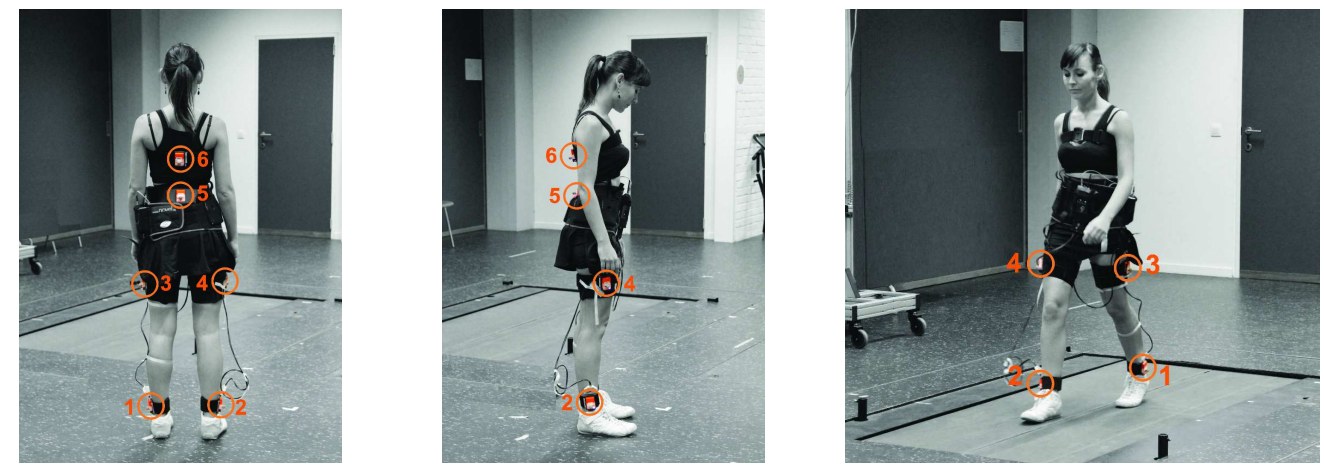

Figure 3: Configuration setup of the wireless inertial units (MTw's) in laboratory conditions.

The accelerations registered near the ankles of the test-subject are assumed to be very strong due to the large motion of these body segments during impact and swing of the leg. Contrary to these sensors that reflect the step-by-step actions of the left or right leg, the sensors on the back gather information about the global walking behaviour of the test-subject. Analysis of the tracked motion and the corresponding measured ground reaction forces will reveal which body segments best display the main characteristics of the walking behaviour.

\subsection{Ground reaction forces}

Figure 4 presents an example of two successive steps registered by the force plates and the corresponding amplitude spectrum of a single step. This figure illustrates that the force spectrum is mainly found below $10 \mathrm{~Hz}$. Figure 5 presents the step-by-step ground reaction forces measured by the instrumented treadmill. To minimise the influence of instrumental noise and moving parts of the treadmill [18], the original signal is decimated by a factor of ten and a cut-off frequency of $12 \mathrm{~Hz}$ is applied (figure 5b) using an eighth order Chebyshev type I filter. Analysis showed no significant difference in the pattern of the forces monitored via the treadmill and the overground trials. As observed by White et al. [19], the force during mid stance is slightly higher and the peak force through late stance is slightly lower for treadmill gait.

\subsection{Analysis of treadmill and overground walking}

In this section, the registered accelerations of the selected body segments are studied in relation to the measured ground reaction forces. Figures $6 \mathrm{a}$ and $6 \mathrm{~b}$ show that the amplitude spectrum of the continuous vertical foot forces (sequence of the single foot forces) and the corresponding vertical acceleration levels at the lower back are highly similar. The frequency of the first dominant peak in these spectra represents the average step frequency of the test-subject during the treadmill trial. Analysis of the registered GRF's and lower back accelerations of the different trials, shows that the same step frequency is in this way identified up to $\pm 0.1 \%$.

Secondly, the onset of each step is derived from the registered time history of the vertical single foot forces and is defined as the time at which $15 \%$ of the peak force is exceeded. The objective is to identify this onset based on the registered motion of the subject. Figures 7 and 8 present the ground reaction forces, the acceleration levels registered at the lower back and the magnitude of the total acceleration vector measured near the ankles. The signals are normalised 
(a)

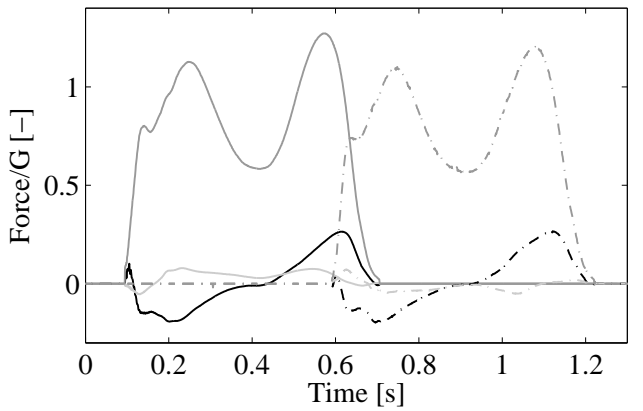

(b)

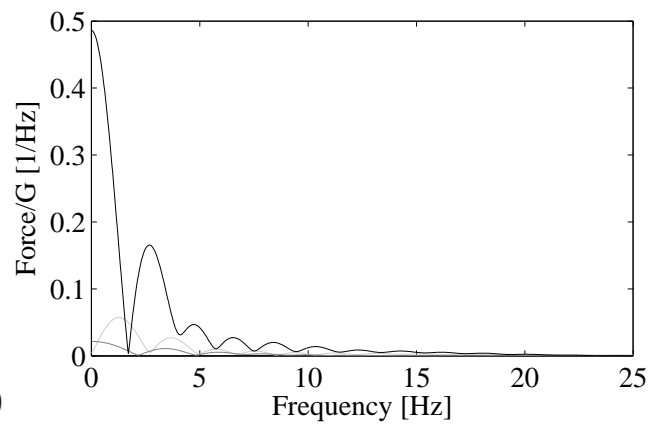

Figure 4: (a) Ground reaction forces registered by the force plates with a sampling frequency of $1 \mathrm{kHz}$ for the left $(-)$ and right (--) foot respectively, normalised to the weight of the test-subject $G$, for a self-selected walking speed and (b) corresponding linear spectrum of a single step: anteroposterior (black), mediolateral (light grey) and vertical (dark grey) component.

(a)

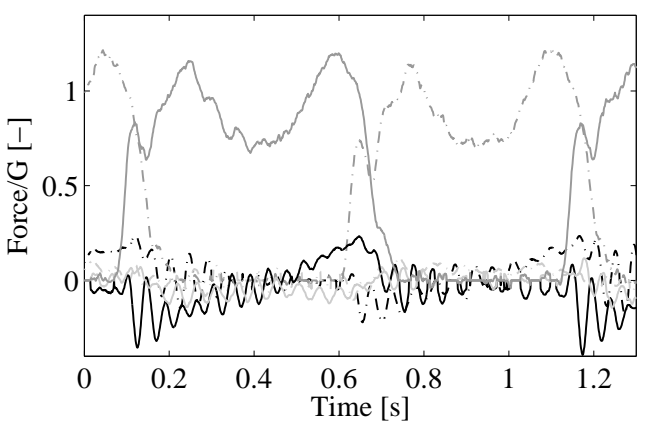

(b)

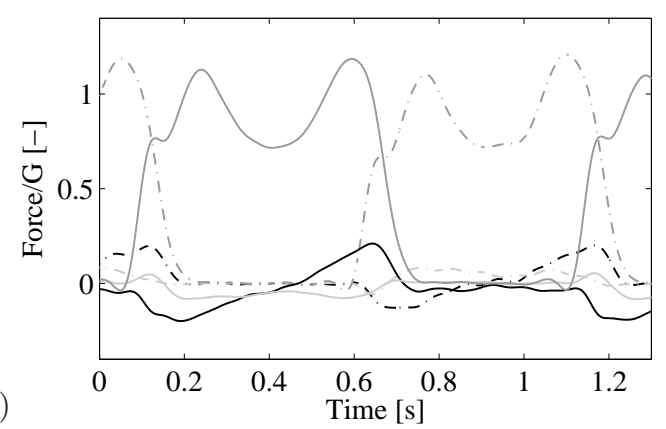

Figure 5: Ground reaction forces for a walking speed of $5 \mathrm{~km} / \mathrm{h}$ for the left (-) and right (-) foot respectively, registered by the instrumented split-belt treadmill: (a) original signal with a sampling frequency of $1 \mathrm{kHz}$ and (b) decimated by a factor of ten and low-pass filtered with a cut-off frequency of $12 \mathrm{~Hz}$ : anteroposterior (black), mediolateral (light grey) and vertical (dark grey) component.

to the weight of the test-subject, the gravitational constant and the mean peak value respectively. In this regard, the onset of each step is defined with the occurrence of a peak in the acceleration levels registered near the ankles of the test-subject (figures $7 \mathrm{e}$ and $7 \mathrm{f}$ ). The same onset, with a certain shift in time, can be identified from the vertical accelerations registered at the lower back (figures $7 \mathrm{c}$ and $7 \mathrm{~d}$ ). Analysis of the different trials shows that this shift is nearly constant for a given step frequency. The same results are confirmed for overground walking (figure 8). The peaks in the acceleration levels registered at the lower back during overground walking are slightly more pronounced which is due to the lower force during mid stance and the higher peak force through late stance in comparison to treadmill walking [19]. This allows for a more accurate identification of the walking behaviour.

Subsequently, the difference between the onsets derived from the registered forces and the onsets identified based on the registered motion of the subject is examined and the corresponding standard deviation from each trial is calculated. Table 1 presents the computed confidence intervals. This table shows that the analysis of the motion registered at the lower back provides the most accurate estimation. The results also show that the accuracy increases with increasing walking speed, which 
(a)

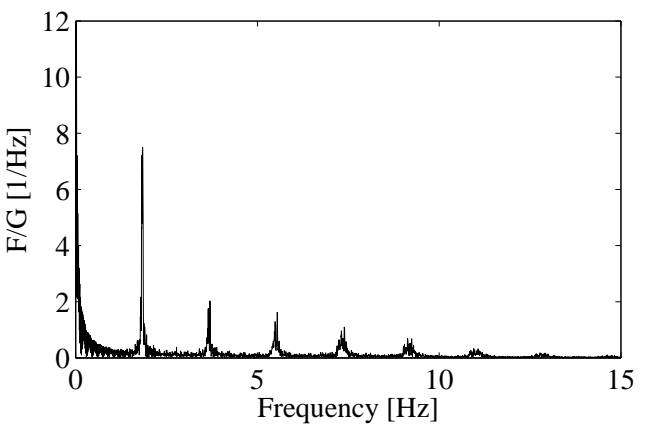

(b)

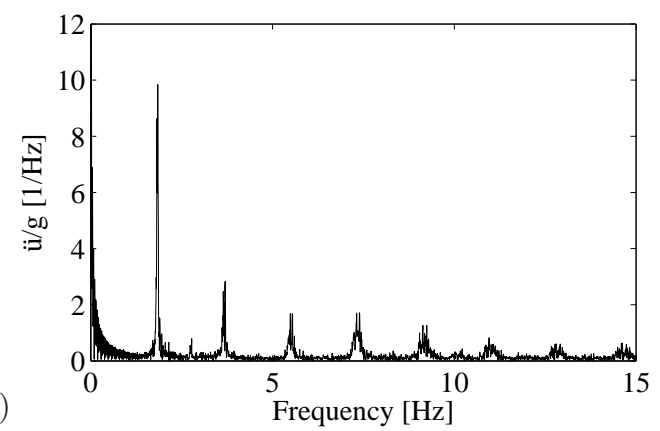

Figure 6: Linear spectrum up to $15 \mathrm{~Hz}$ of (a) the vertical ground reaction forces (sum of left and right foot) registered by the instrumented split-belt treadmill for a walking speed of $5 \mathrm{~km} / \mathrm{h}$ and (b) the corresponding measured vertical acceleration levels at the lower back.

is due to the corresponding reduced contact time of each step. For a normal walking speed of 1.85 $\mathrm{Hz}$, the onset of each step can be identified with $95 \%$ accuracy up to 0.01 seconds.

\begin{tabular}{ccccc}
\hline $\begin{array}{c}\text { walking speed } \\
{[\mathrm{km} / \mathrm{h}]}\end{array}$ & $\begin{array}{c}\text { step frequency } \\
{[\mathrm{Hz}]}\end{array}$ & $\begin{array}{c}\text { \# steps } \\
{[-]}\end{array}$ & $\begin{array}{c}\text { lower back } \\
2 \sigma[\mathrm{s}]\end{array}$ & $\begin{array}{c}\text { ankles } \\
2 \sigma[\mathrm{s}]\end{array}$ \\
\hline 3.0 & 1.55 & 166 & 0.018 & 0.031 \\
3.5 & 1.68 & 178 & 0.014 & 0.032 \\
4.0 & 1.75 & 182 & 0.012 & 0.022 \\
4.5 & 1.85 & 193 & 0.011 & 0.017 \\
5.0 & 1.92 & 200 & 0.011 & 0.017 \\
5.5 & 2.00 & 215 & 0.010 & 0.018 \\
6.0 & 2.06 & 217 & 0.010 & 0.016 \\
\hline
\end{tabular}

Table 1: Summary of the different walking speeds, the mean step frequency, the number of registered steps and the $95 \%$ confidence interval of the identified onset of each step based on the motion registered at the lower back and near the ankles, for each trial. 
(a)

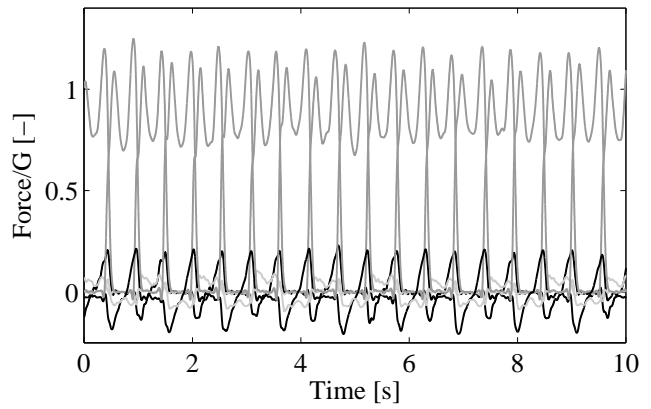

(b)

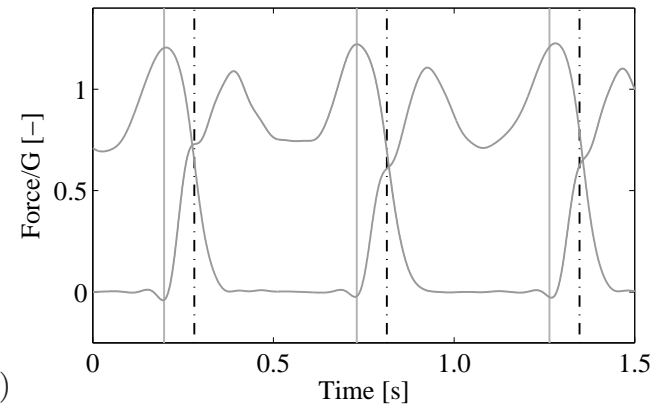

(c)

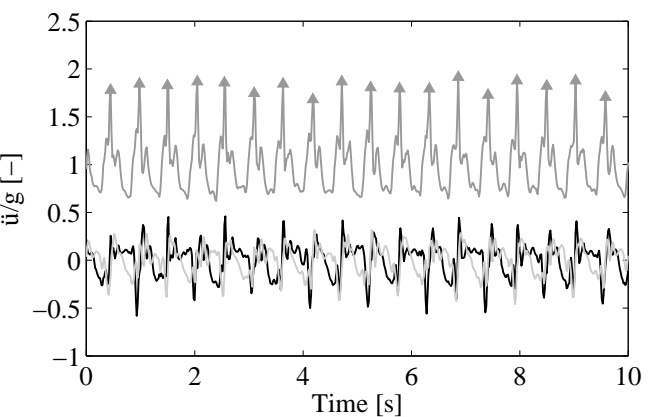

(d)

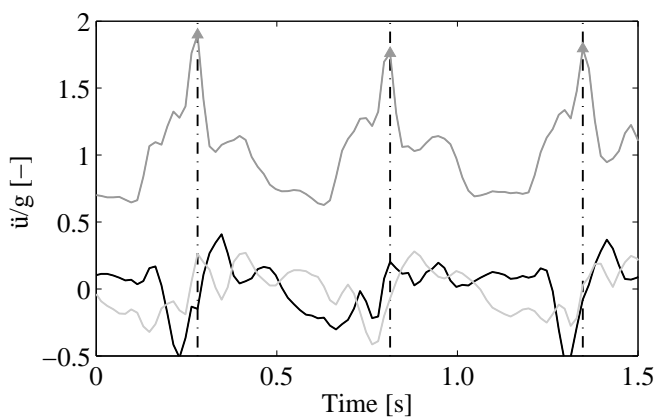

(e)

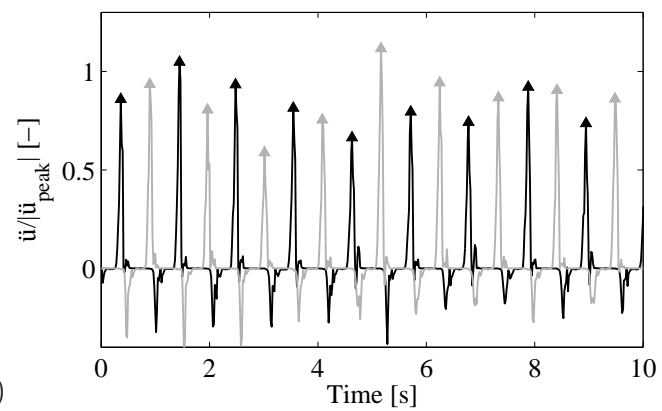

(f)

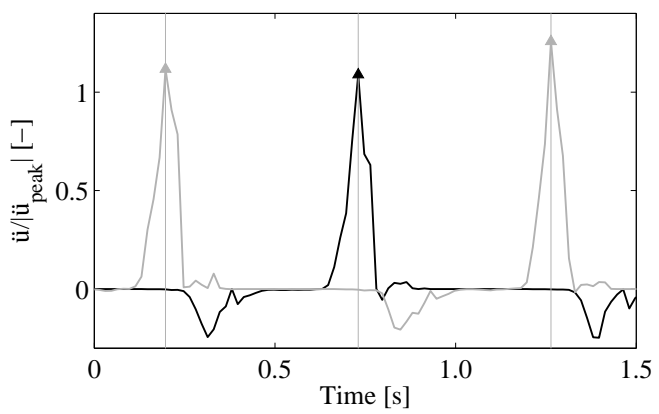

Figure 7: For a walking speed of $5 \mathrm{~km} / \mathrm{h}$ : (a-b) normalised ground reaction forces registered by the instrumented split-belt treadmill, (c-d) normalised measured accelerations at the lower back for the longitudinal (black), lateral (light grey) and vertical (dark gray) component and (e-f) normalised magnitude of the total acceleration vector measured near the left (black) and right (gray) ankle. 
(a)

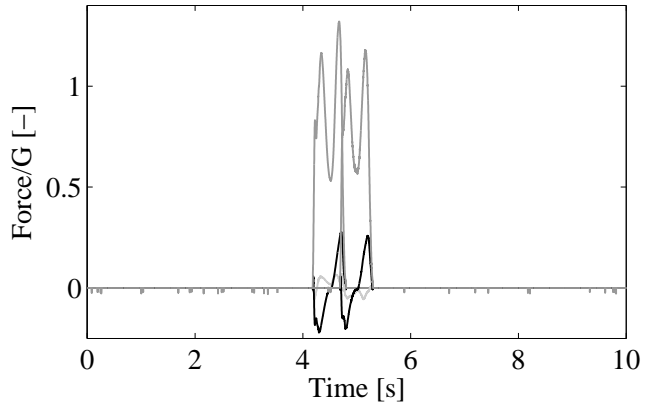

(c)

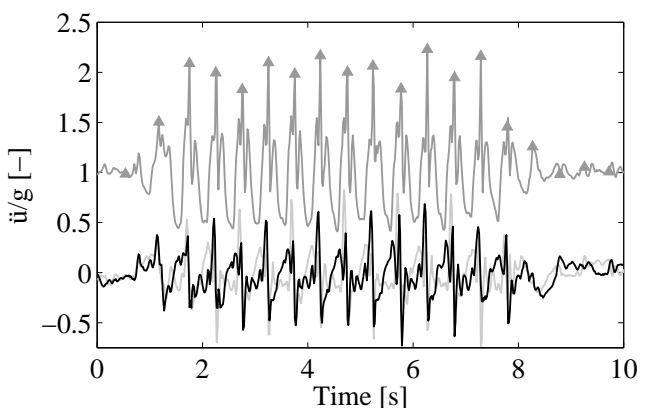

(d)

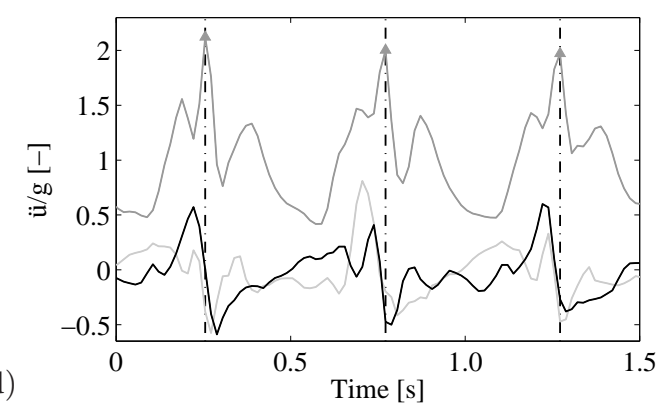

(e)

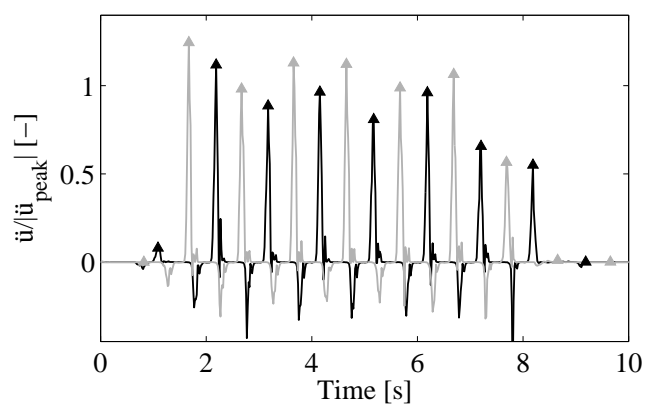

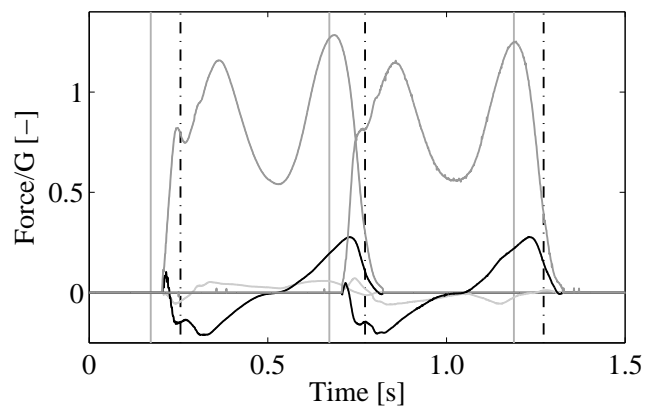

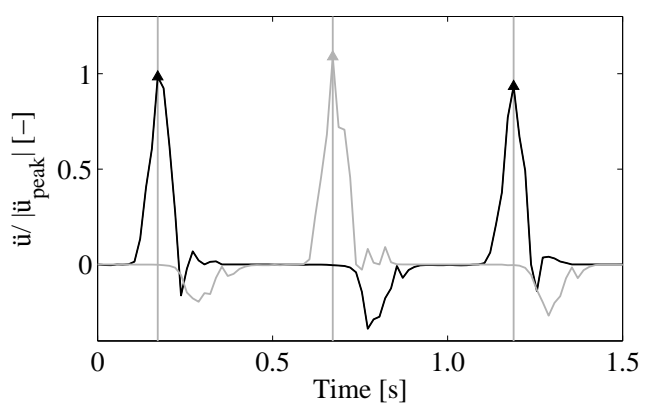

Figure 8: For a normal walking speed: (a-b) normalised ground reaction forces registered by the force plates, (c-d) normalised measured accelerations at the lower back for the longitudinal (black), lateral (light grey) and vertical (dark gray) component and (e-f) normalised magnitude of the total acceleration vector measured near the left (black) and right (gray) ankle. 


\subsection{Simulation of the ground reaction forces}

In this section, it is verified if the measured vertical ground reaction forces can be accurately simulated using a step-by-step load model developed by Li et al. [20] for which the characteristics are determined based on the registered motion of the body segments. To include stride-to-stride variations, the results of the treadmill experiments are used.

First, the mean step frequency of the test-subject for the considered treadmill trial is determined based on the frequency domain analysis of the accelerations measured at the lower back. This step frequency $f_{s}[\mathrm{~Hz}]$ along with the static weight of the test-subject $G[\mathrm{~N}]$, determine the vertical single foot force $F_{1}[\mathrm{~N}]$ as defined by Li et al. [20]:

$$
F_{1}(t)=G \sum_{n=1}^{5} A_{n} \sin \left(\frac{\pi n}{T_{c}} t\right), \quad 0 \leq t \leq T_{c}
$$

with $A_{n}[-]$ the Fourier coefficient, normalised to the weight of the pedestrian, and $T_{c}[\mathrm{~s}]$ the duration of the contact between the foot and the ground, both defined as a function of the step frequency $f_{s}$ by Li et al. [20] for different ranges of $f_{s}$. The relation between the step frequency and contact duration is relatively insensitive to different walking speeds [21]:

$$
T_{c}=\frac{1}{0.76 f_{s}}
$$

Secondly, the registered motion is applied to determine the onset of each step in time $\left(t_{i}\right)$. Subsequently, the corresponding force due to step $i$ in time is found as:

$$
F_{i}(t)=\kappa\left(t-t_{i}\right) F_{1}\left(t-t_{i}\right), \quad \text { with } \quad \kappa(t)= \begin{cases}1 & 0 \leq t \leq T_{c} \\ 0 & \text { otherwise }\end{cases}
$$

with $t[\mathrm{~s}]$ the general time of the considered treadmill trial. Together, the mean step frequency and onset of each step, provide the necessary input to simulate the measured ground reaction forces based on the generalised vertical single foot force of Li et al. [20].

Figure 9 shows that there is a small difference between the simulated vertical single foot force of Li et al. [20] and the measured forces. This difference could be minimised by replacing $F_{1}(t)$ by the averaged vertical single foot force of the test-subject for the corresponding walking speed. Since the direct force measurements are not available for the test-subjects involved in the in-situ experiments discussed in the next section, it is preferred to also apply the generalised force model here. Moreover, it is expected that the simulated vibration response is more sensitive to variations of the pacing rate for successive footfalls than to small variations in force amplitude or contact time, as observed by Middleton [22]. Figure 9 illustrates that the onset of each step, and therefore also the pacing rate, is accurately identified from the registered motion of the test-subject.

Figure 10 presents the amplitude spectrum of the simulated and measured ground reaction forces. In contrast to perfectly periodic forces that are exclusively composed of the harmonics of the step frequency, imperfect real walking results into a distribution of forces around the dominant harmonics $[8,23]$. Figure 10 illustrates that these narrow band forces are clearly present in the measured forces but also in the simulated forces that account for the identified pacing rate. This comparison shows that simulations based on the generalised single foot force model and the in situ identified pacing rate, allow for a good approximation of the GRF's in case of imperfect real walking. 

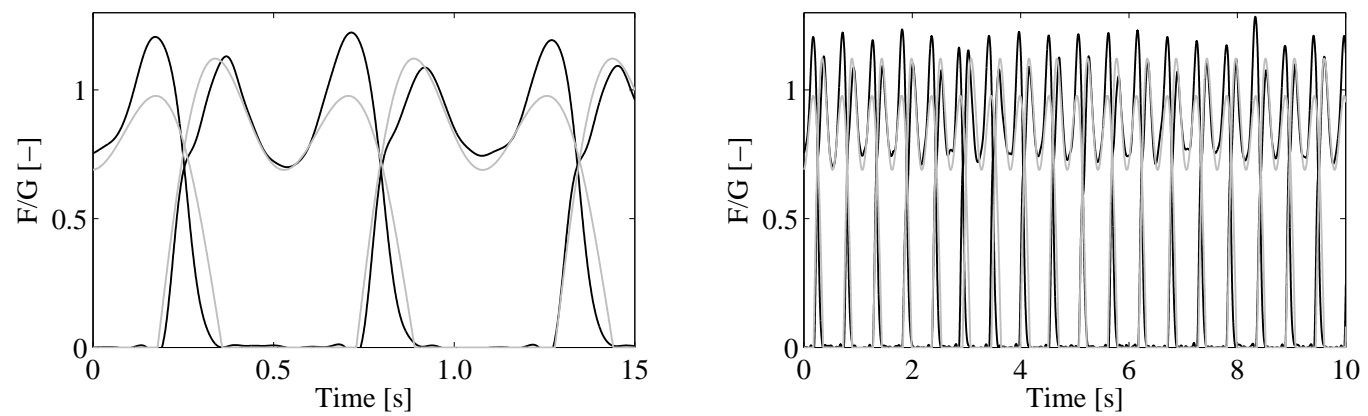

Figure 9: The measured vertical ground reaction forces for a walking speed of $5 \mathrm{~km} / \mathrm{h}$ (black) and the corresponding simulated vertical walking forces with step frequency and time in between two successive steps identified based on the tracked motion of the body segments of the test-subject (grey).

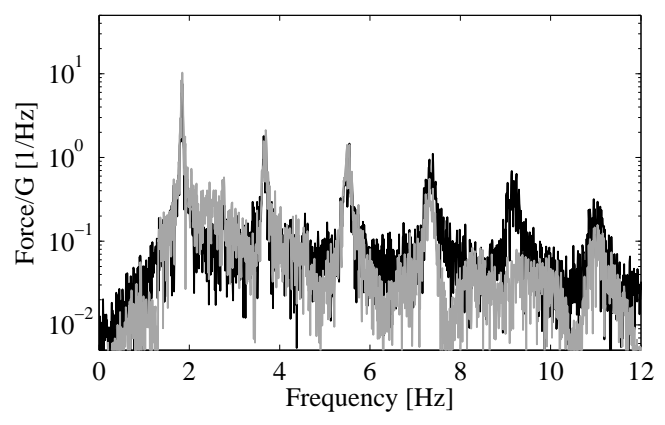

Figure 10: Linear spectrum of the measured vertical ground reaction forces for a walking speed of $5 \mathrm{~km} / \mathrm{h}$ (black) and the corresponding simulated vertical walking forces according to Li et al. [20] with mean step frequency $f_{s}$ and pacing rated identified based on the tracked motion of the body segments of the test-subject (grey).

\section{Application in situ}

This section discusses the in situ experiments performed on a lightweight steel footbridge. The motion of the pedestrians and the structural response are registered simultaneously. Prior to these experiments, ambient vibration measurements have been performed to identify modal characteristics of the footbridge. The identified natural frequencies and mode shapes are applied to calibrate the numerical model of the structure. Additional free decay tests are performed to study the effect of the presence of the standing crowd onto the modal damping ratios. Numerical simulations are performed applying step-by-step force models and the impact of the in situ identified imperfect walking behaviour of the test-subjects is assessed.

\subsection{Eeklo footbridge}

The footbridge considered in these experiments is located in Eeklo, at Aalstgoed, and forms a safe passage across the N49 for pedestrians and cyclists. The bridge (2002) has three spans, a main central span of 42 meter (across the N49) and two side spans of 27 meter (figure 11a). The bridge is simply supported with land abutments at the sides and two piers at the center span. The abutments and piers are equipped with neoprene supports.

The cross section of the bridge (figure 11b) consists of two main beams with a height of 1.2 meter at a spacing of 3.4 meter, supporting a steel deck of 8 millimeters thickness via three secondary 
(a)

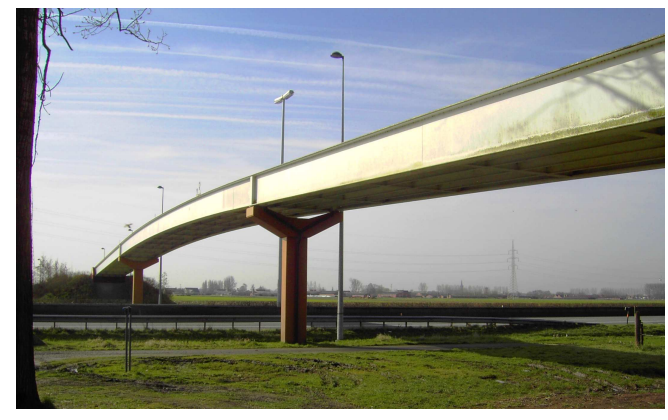

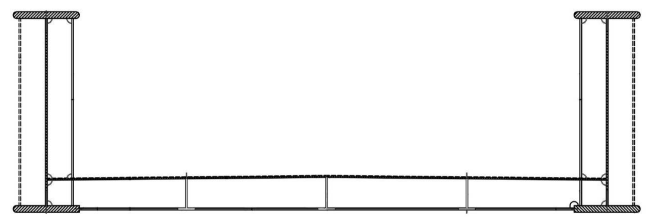

(b)

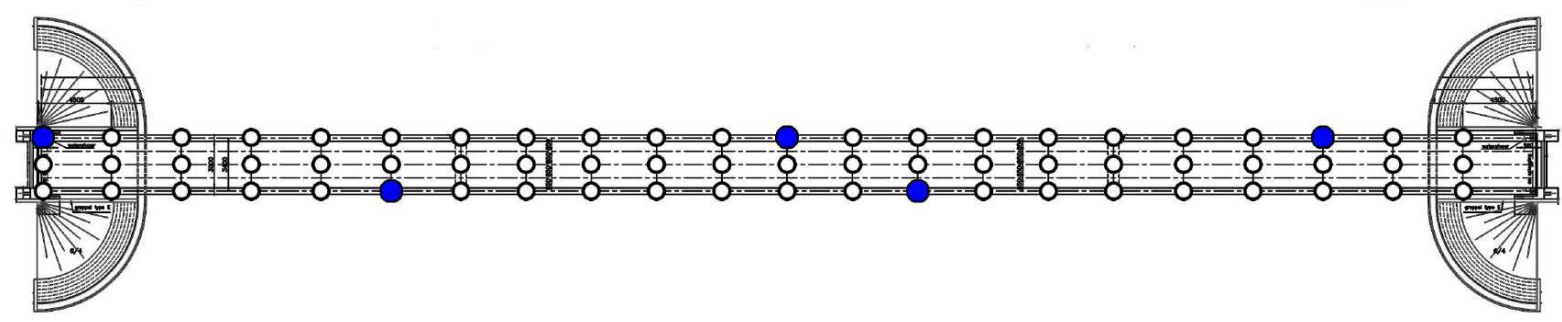

Figure 12: Plan view of the Eeklo footbridge including the location of the roving and reference sensors (dark).

beams. At midspan of the bridge, horizontal and vertical transverse beams are positioned at a distance of 4.2 meter and at the side spans at a distance of 4.5 meter. The two side spans have an inclination of 5\% and the midspan traces a curve (circle segment) with a radius of 420 meter.

The following two paragraphs discuss respectively the finite element model updating and the experimental modal damping estimation under human occupation.

\subsubsection{Finite Element Model Updating}

An extensive measurement campaign was carried out to obtain the operational modal characteristics of the footbridge. Output-only system identification is performed based on ambient vibrations, mainly due to wind and nearby highway traffic $[24,25,26]$. In total, 14 modes were identified with a frequency up to $12 \mathrm{~Hz}$ (table 2). Some of the lower operational eigenmodes and eigenfrequencies identified by this analysis, are presented in figure 13 which illustrates that combined lateral-torsional modes alternate with vertical bending modes.

The FE model was developed in great detail according to the as-built plans. The model of the structure consists of Timoshenko beam elements for the longitudinal and transversal stiffeners and a regular mesh of 4-node shell elements (Mindlin-Reissner theory) with six degrees of freedom at each node for the steel plates fixed to the stiffeners. The concrete piers consist of solid elements and the neoprene bearings are modelled with translational springs. This leads to a system with a total number of 44757 degrees of freedom (DOF).

The results of the operational modal analysis were applied to calibrate the FE model: model parameters are adopted such that an optimal correspondence is found between measured and 


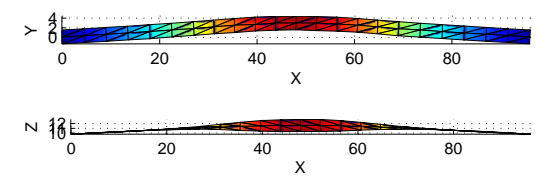

(a)

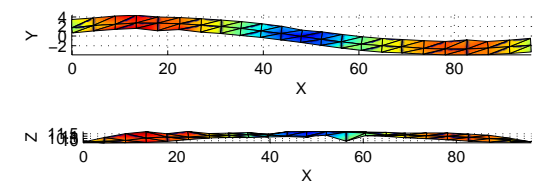

(d)

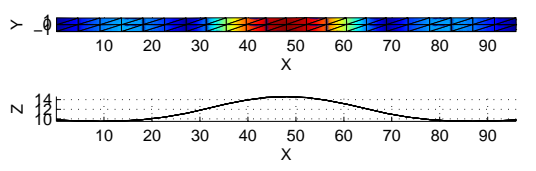

(b)

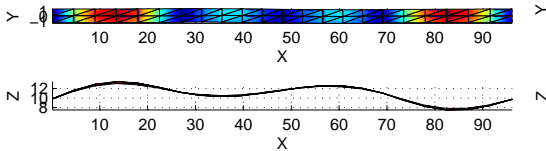

(e)

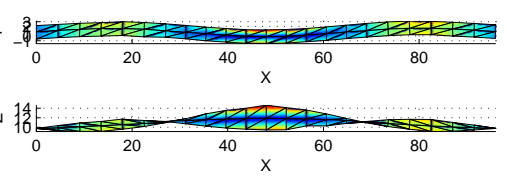

(c)

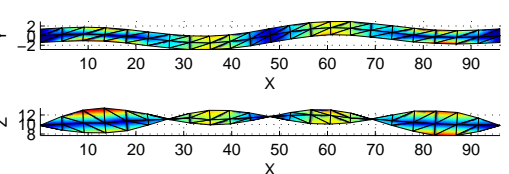

(f)

Figure 13: Top and side view of the first six identified modes of the Eeklo footbridge: (a) Mode 1 with $\tilde{f}_{1}=1.71 \mathrm{~Hz}$, (b) Mode 2 with $\tilde{f}_{2}=2.99 \mathrm{~Hz}$, (c) Mode 3 with $\tilde{f}_{3}=3.25 \mathrm{~Hz}$, (d) Mode 4 with $\tilde{f}_{4}=3.46 \mathrm{~Hz}$, (e) Mode 5 with $\tilde{f}_{5}=$ $5.77 \mathrm{~Hz}$ and (f) Mode 6 with $\tilde{f}_{6}=5.82 \mathrm{~Hz}$.

calculated modal characteristics. This results in a better representation of the dynamic behaviour of the structure and, therefore, increases the accuracy of the numerical predictions of the response to pedestrian loading. The updating variables are those parameters for which poor prior knowledge is available. Due to the simplicity of the structure of the footbridge, only one type of updating variable is considered in this analysis: the stiffnesses of the neoprene supports. In the FE model, these bearings are modelled with three translational springs (one in vertical, lateral and longitudinal direction) at each support.

Table 2 summarises the results of the updating procedure. It lists the values of the measured natural frequencies $\tilde{f}$, calculated natural frequencies $f$, the relative error $\varepsilon_{j}$, and MAC values between measured and calculated mode shapes. This table shows that an excellent agreement is found between the predicted and identified modal characteristics of the bridge. The relative errors on the frequencies of all modes are limited to $1.76 \%$ and the MAC values are all close to 1 . Three modes predicted by the calibrated FE model are not identified in the operational modal analysis due to the limited modal displacements of the bridge deck. They relate to a quasi rigid body mode of the bridge deck (mode 1) and two bending modes of the piers (mode 12 and 13).

\subsubsection{Influence of human occupation on the structural dynamic properties}

There is a general agreement that an active or passive crowd interacts with the supporting structure $[27,28]$. For footbridges exhibiting significant lateral movements, the interaction between the pedestrian and the structure, potentially results into additional non-negligible forces $[29,10]$. In case of dominant vertical vibrations, the observed interaction is mainly reflected by changes in structural dynamic properties but the interaction itself is far less understood [28, 30].

The Eeklo footbridge is a slender structure with low inherent damping and mode shapes with large vertical displacements. It is expected that the crowd-structure interaction will for the most part result into changes in dynamic properties as compared to the empty structure. Since the following section considers resonant conditions for the first two modes of the structure, the critical parameters are the modal damping ratios of the new human-structure system. These damping ratios are expected to increase in presence of pedestrians on the structure [30,31].

The modal damping ratios of the first and the second mode are identified experimentally by means of a free vibration test. To analyse their sensitivity to the presence of the pedestrians, the 


\begin{tabular}{rrrrrrc}
\hline$\tilde{N}$ & $\tilde{f}[\mathrm{~Hz}]$ & $\tilde{\xi}[\%]$ & $N$ & $f[\mathrm{~Hz}]$ & $\varepsilon[\%]$ & MAC $[-]$ \\
\hline 1 & 1.71 & 1.94 & 2 & 1.71 & 0.26 & 0.99 \\
2 & 2.99 & 0.19 & 3 & 3.02 & 0.90 & 1.00 \\
3 & 3.25 & 1.45 & 4 & 3.30 & 1.76 & 0.98 \\
4 & 3.46 & 2.97 & 5 & 3.43 & -0.67 & 0.98 \\
5 & 5.77 & 0.23 & 6 & 5.75 & -0.31 & 0.99 \\
6 & 5.82 & 0.16 & 7 & 5.80 & -0.46 & 0.97 \\
7 & 6.04 & 2.08 & 8 & 6.10 & 0.89 & 0.97 \\
8 & 6.47 & 0.60 & 9 & 6.47 & 0.00 & 1.00 \\
9 & 6.98 & 3.38 & 10 & 6.94 & -0.48 & 0.96 \\
10 & 7.44 & 4.77 & 11 & 7.36 & -1.09 & 0.97 \\
11 & 9.64 & 0.87 & 15 & 9.80 & 1.67 & 1.00 \\
12 & 9.89 & 2.50 & 14 & 9.71 & -1.76 & 0.96 \\
13 & 10.48 & 1.43 & 16 & 10.65 & 1.62 & 0.97 \\
14 & 12.01 & 3.49 & 17 & 12.16 & 1.30 & 0.93 \\
\hline
\end{tabular}

Table 2: Identified modal characteristics (mode number $\tilde{N}$, natural frequencies $\tilde{f}$, damping ratios $\tilde{\xi}$ ) of all modes with a natural frequency below $13 \mathrm{~Hz}$ and corresponding mode number $N$ and natural frequencies $f$ predicted by the calibrated numerical model, the calculated Modal Assurance Criterion (MAC) and the relative frequency deviation $\varepsilon$.

experiments include setups with a different number of test-subjects on the bridge deck. Each of the test-subjects is positioned on top of a crossing of a secondary beam with a transversal beam (in agreement with the sensor setup presented in figure 12), starting with three persons at midspan. For each of the following setups, the number of test-subjects is increased by six, three on the left and three on the right adjacent transversal beam respectively. For the setups focused on the fundamental torsional mode, the test-subjects on the central longitudinal axis are omitted. As a second element, the influence of the posture of the pedestrians is examined. This posture is changing during the walking cycle and is mainly characterised by the angle between upper and lower leg. The experiments therefore consider the human body in different standing postures: straight legs and slightly bent legs.

The free vibrations, dominated by the contribution of a single mode, are induced by the bobbing of the different test-subjects on the structure. Their activities are synchronised using a metronome signal chosen to match the selected natural frequency. Once the desired level of vibration is reached, the test-subjects cease bobbing and get into the selected posture, thereby initiating the free decay.

An exponential function is fitted to the relative maxima of the recorded decay with amplitudes between $90 \%$ and $20 \%$ of the maximum acceleration [32]. From figures $14 \mathrm{~b}$ and $14 \mathrm{e}$, it can be observed that the assumption of the contribution of a single mode is justified. Figures 14c and $14 \mathrm{f}$ present the natural logarithm and the least-squares line approximation of peak amplitudes $\left(y_{i}\right)$ with peak points $\left(x_{i}\right)$. They illustrate that the damping characteristics hardly depend on the vibration amplitudes. Table 3 and 4 resume the results obtained from the exponential fitting for the different setups. These results show that the presence of the test-subjects leads to a significant increase of the modal damping ratios. This increase is, however, more pronounced for the first vertical bending mode, which is due to the low value of the corresponding modal damping ratio for the empty structure. Considering the different postures, no clear trend can be identified for the first mode but for the second mode it is clear that the additional damping due to the presence of the test-subjects with slightly bent legs is significantly larger. The test-subjects reported as if the 


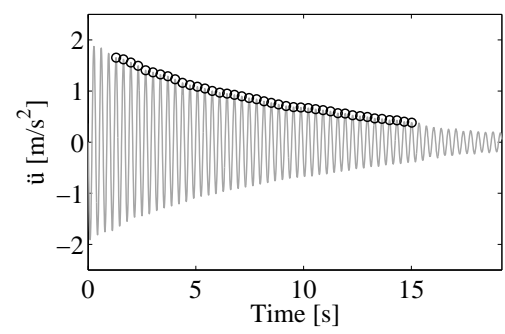

(a)

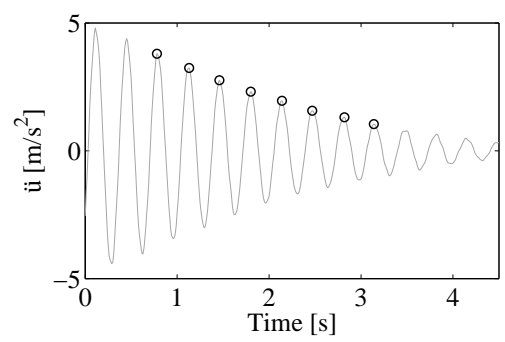

(d)

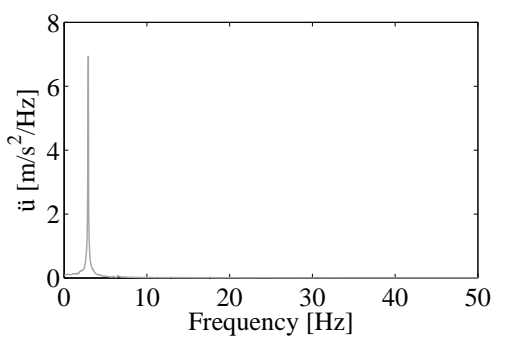

(b)

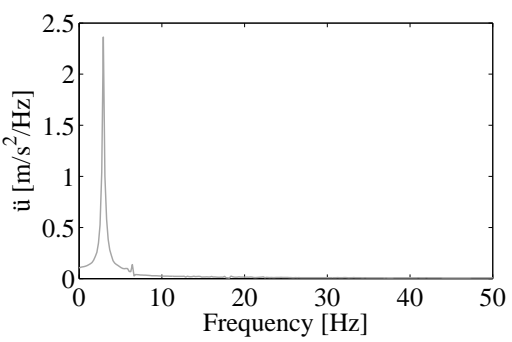

(e)

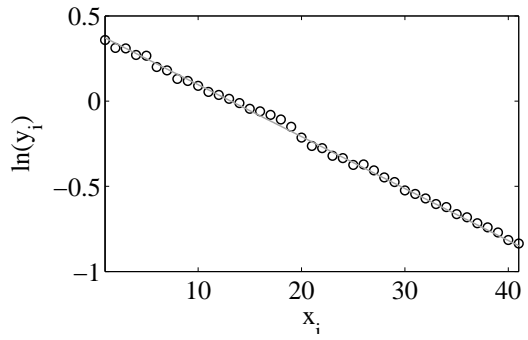

(c)

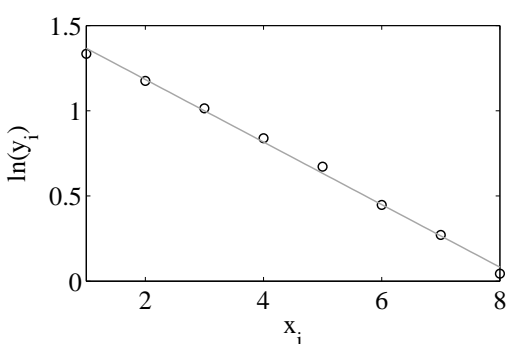

(f)

Figure 14: Measured vertical free vibration response of the footbridge at midspan including the presence of 15 test-subjects (a-c) with straight legs and (d-f) slightly bent legs: (a, d) time series with selected extremes, (b, e) corresponding amplitude spectrum and (c, f) natural logarithm and best-fit line of peak amplitudes $\left(y_{i}\right)$ with peak points $\left(x_{i}\right)$.

structural vibration was forcing them into an opposite movement.

\subsection{Analysis of the walking behaviour in situ}

Different setups are considered in the experiments including free walking and synchronised walking. In case of synchronised walking, the step frequency (or its second harmonic) of the test-subjects is chosen to match one of the natural frequencies of the structure. These resonant conditions are realised for both the first $\left(f_{s}=\tilde{f}_{1}=1.71 \mathrm{~Hz}\right)$ and the second mode $\left(f_{s}=\tilde{f}_{2} / 2=1.49\right.$ $\mathrm{Hz}$ ) of the structure. The step frequencies of the pedestrians are imposed using a metronome. Prior application in laboratory conditions showed that, despite the use of a metronome, perfect synchronisation is never achieved by the test-subjects.

Different group sizes, ranging from one pedestrian up to six, are considered in various formations: two by two or all lined up one by one, and symmetrically (figure 15a) or asymmetrically (figure 15b) distributed with respect to the longitudinal axis of the structure.

The response of the structure is registered with five triaxial sensors (reference sensors in figure 12), for which the location was determined using an optimal sensor location algorithm [33]. The walking behaviour of the pedestrians is registered with the 3D motion tracking technique. Due to the limited number of MTw's, a single sensor is fixed onto each test-subject and is positioned close to his center of mass.

The identification of the pacing rate in situ is illustrated for a setup where the second harmonic of the walking load is set to match the frequency of the second mode of the structure $\left(f_{s}=\tilde{f}_{2} / 2=\right.$ $1.49 \mathrm{~Hz}$ ). At a walking speed of $1.2 \mathrm{~m} / \mathrm{s}$, it takes about 90 seconds for all six test-subjects to cross the footbridge. Figure $16 \mathrm{~b}$ presents the accelerations measured close to the COM of the 


\begin{tabular}{ccccc}
\hline \# test- & \multicolumn{2}{c}{ straight legs } & \multicolumn{2}{c}{ bent legs } \\
subjects & $\tilde{f}_{1}[\mathrm{~Hz}]$ & $\tilde{\xi}_{1}[\%]$ & $\tilde{f}_{1}[\mathrm{~Hz}]$ & $\tilde{\xi}_{1}[\%]$ \\
\hline empty structure & $\mathbf{1 . 7 1}$ & $\mathbf{1 . 9 4}$ & & \\
2 & 1.68 & 2.64 & 1.70 & 2.16 \\
6 & 1.66 & 2.75 & 1.68 & 2.52 \\
10 & 1.67 & 2.61 & 1.66 & 2.68 \\
14 & 1.66 & 2.66 & 1.66 & 2.59 \\
18 & 1.66 & 2.53 & 1.64 & 2.93 \\
\hline
\end{tabular}

Table 3: Identified natural frequency $\tilde{f}_{1}$ and modal damping ratio $\tilde{\xi}_{1}$ of the first combined lateral-torsional mode, provided by the exponential fitting of the corresponding free decay.

\begin{tabular}{ccccc}
\hline \# test- & \multicolumn{2}{c}{ straight legs } & \multicolumn{2}{c}{ bent legs } \\
subjects & $\tilde{f}_{2}[\mathrm{~Hz}]$ & $\tilde{\xi}_{2}[\%]$ & $\tilde{f}_{2}[\mathrm{~Hz}]$ & $\tilde{\xi}_{2}[\%]$ \\
\hline empty structure & $\mathbf{2 . 9 9}$ & $\mathbf{0 . 1 9}$ & & \\
3 & 2.97 & 0.26 & 2.99 & 1.19 \\
9 & 2.94 & 0.44 & 3.00 & 2.02 \\
15 & 2.92 & 0.53 & 2.96 & 3.07 \\
21 & 2.91 & 0.66 & 2.92 & 3.47 \\
26 & 2.90 & 0.61 & 2.92 & 3.41 \\
\hline
\end{tabular}

Table 4: Identified natural frequency $\tilde{f}_{2}$ and modal damping ratio $\tilde{\xi}_{2}$ of the first vertical bending mode, provided by the exponential fitting of the corresponding free decay.

6 test-subjects. For reasons of clarity, the signal of the first pedestrian is emphasised. These registered accelerations are clearly similar to the ones obtained in laboratory conditions presented in figures $7 \mathrm{c}, 7 \mathrm{~d}, 8 \mathrm{c}$ and $8 \mathrm{~d}$. The onset of each step, and thus the pacing rate, is identified for each test-subject applying the methodology described in section 2.4. Time synchronisation among the MTw's allows the synchronisation rate of the different test-subjects to be studied.

In figure 16a, the onset of each step of every test-subject is indicated by a single vertical line. When these lines coincide, the different test-subjects take their steps at more or less the same time and a high rate of synchronisation has been obtained. Lines equally spaced at $1 / f_{s}$ therefore represent a high synchronisation rate. From figures 16a and 16b it can be observed that for this setup, the rate of synchronisation is relatively high during the first 40 seconds. Rather scattered lines on the other hand, indicate a lower synchronisation rate. Figures 16a and 16c illustrate that the synchronisation is lost between 50 and 60 seconds and gets partially restored towards the end.

As discussed in section 2.5, the induced forces can be simulated from the in situ identified pacing rate and the generalised single foot force model that accounts for the identified mean step frequency and the weight of every test-subject. Figures 16d and 16e present the simulated forces for all test-subjects corresponding to the registered motion presented in figures 16b and 16c. These figures again clearly depict a period in time characterised by a high and a low synchronisation rate, respectively.

\subsection{Numerical simulations}

The essential components of the simulation model are the dynamic behaviour of the footbridge, the dynamic footfall load and the response calculation. The dynamic behaviour of the footbridge is characterised by the modal parameters previously discussed in section 3.1. In the calculations the natural frequencies and mode shapes of the calibrated finite element model and the in situ 
(a)

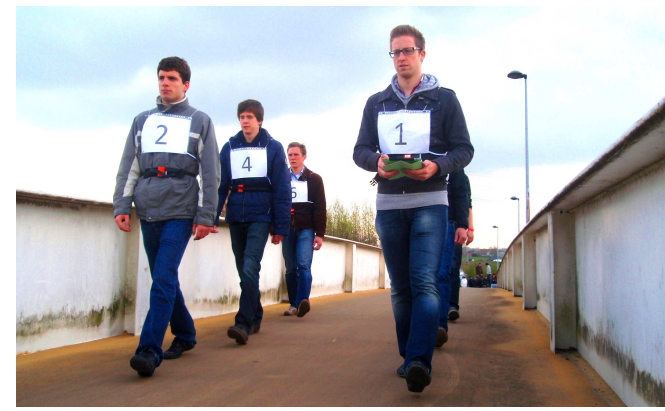

(b)

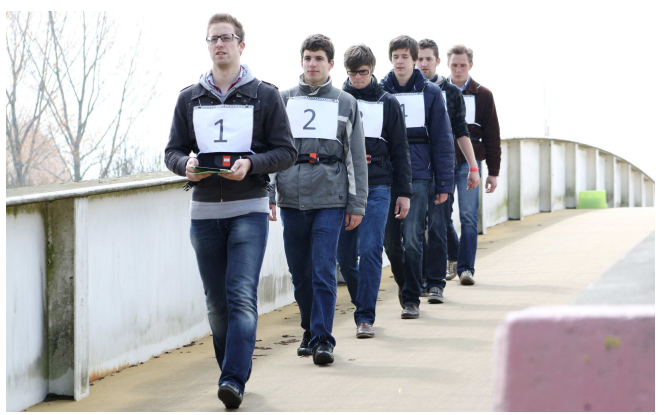

Figure 15: Synchronised walking of 6 persons (a) two by two and (b) in a row.

identified modal damping factors are used. All modes with a natural frequency below $20 \mathrm{~Hz}$ are taken into account.

In these numerical simulations the damping ratio of the bare structure is used, disregarding the influence of the presence of the pedestrians. This influence depends on both posture and location, changing as the pedestrian crosses the bridge. Further research is needed to allow for a proper assessment of the impact of the presence of pedestrians. The comparison with the registered structural response will indicate whether or not pedestrian-structure interaction is significant for the considered experiments.

The walking forces are simulated as a series of moving single foot forces. The vertical component is described by $\mathrm{Li}$ et al. [20], as discussed in section 2.5. The lateral component is described by a piecewise constant force with an alternating sign for left and right foot and with a contact time equal to the vertical force. Its amplitude is set to 0.04G, as recommended by Butz et al. [7].

The response is calculated by time integration of the state-space equations formulated in terms of the modal coordinates.

Figure 17 presents the measured and simulated acceleration response at midspan for the setup where the subjects are walking on one side of the bridge, with a pacing rate targeted at $\tilde{f}_{1}$. This figure presents the lateral response at midspan, which is the dominant component of the first mode. In total, 3 different results are presented: the simulated acceleration response due to (1) perfectly periodic forces based on the identified mean step frequency of the test-subjects, (2) the imperfect near-periodic walking forces accounting for the identified mean step frequency and onset of each step and (3) the registered response. This figure illustrates that the response of the structure is overestimated by the perfectly periodic forces. Although accounting for imperfect walking behaviour by applying the identified pacing rate still leads to an overestimation of the response, a much better qualitative agreement is found with the measured response. For both this simulated and measured response, a decrease in acceleration level can be observed after about 40 seconds, which is due to a reduced synchronisation of the test-subjects. The comparison in the frequency domain (figure 17-b) confirms that accounting for the imperfect walking behaviour results into a better agreement with the measured response. The underestimation at low frequencies is mainly due to ambient excitation of the bridge due to wind and nearby traffic, which is unaccounted for in this analysis. The discrepancy between the predicted an measured response at higher frequencies is mainly due to modelling errors still present in the calibrated finite element model.

Figure 18 presents the measured and simulated vertical acceleration response at midspan for 


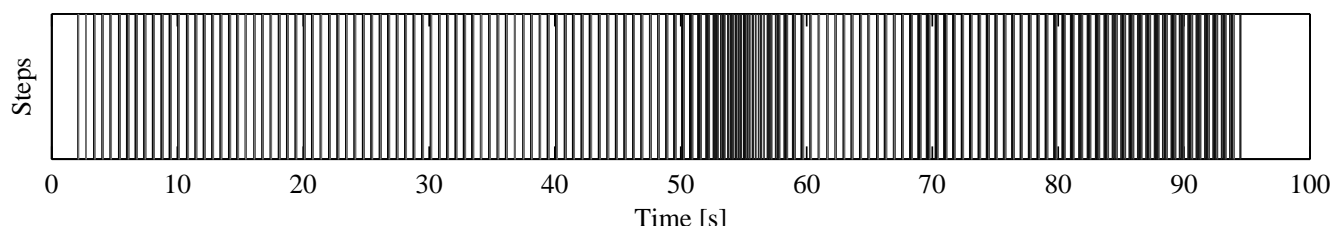

(a)

Time $[\mathrm{s}]$

(b)

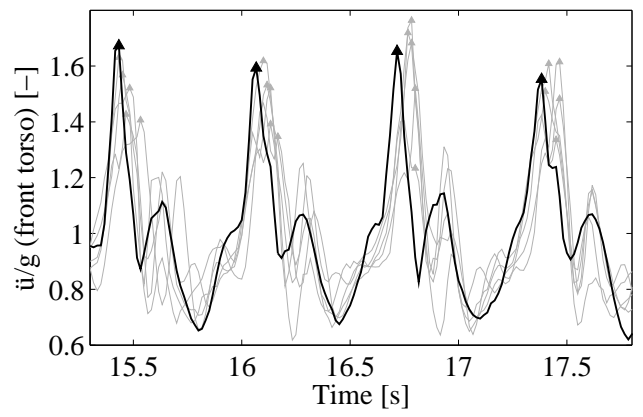

(c)
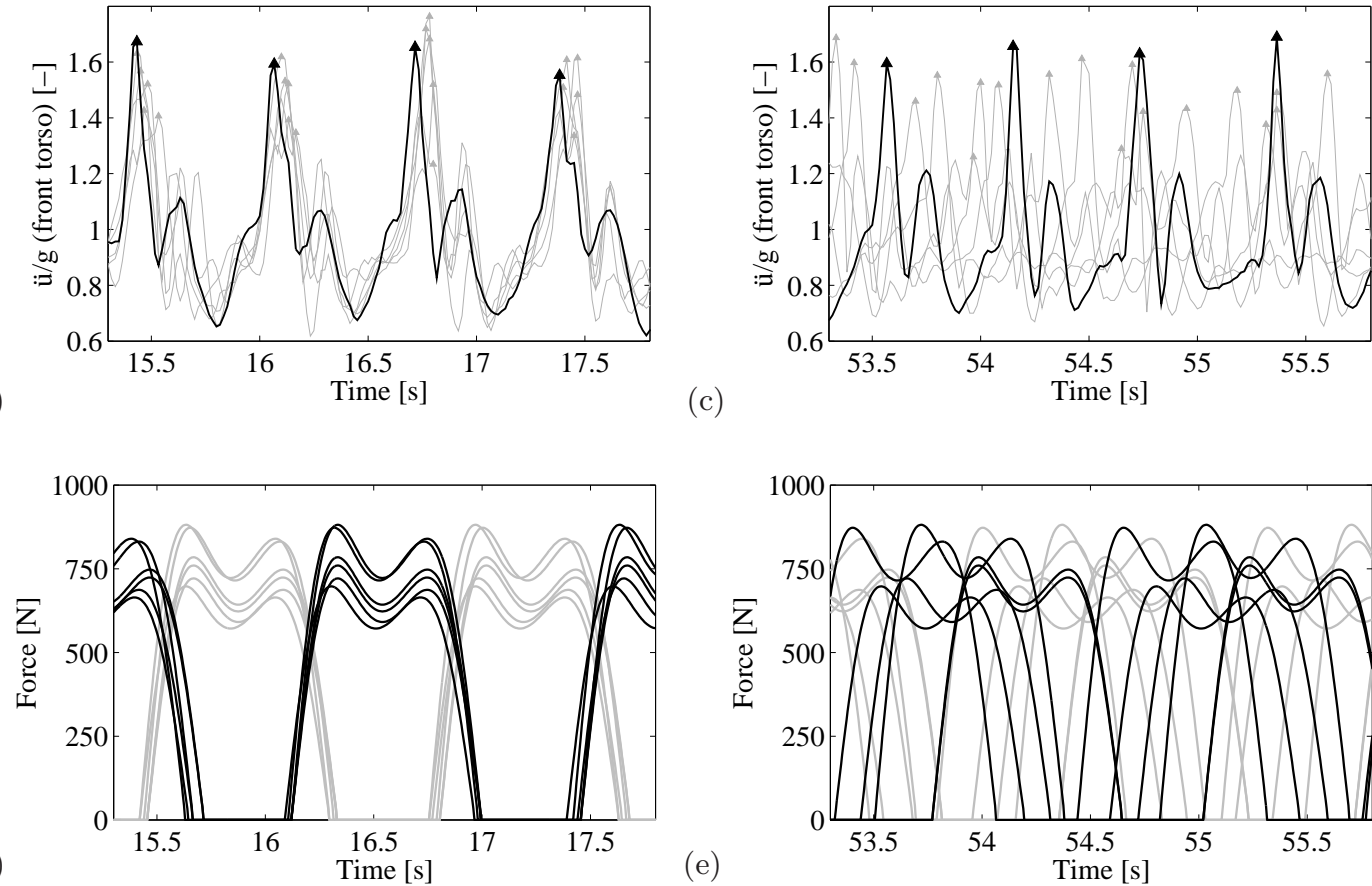

$(\mathrm{e})$

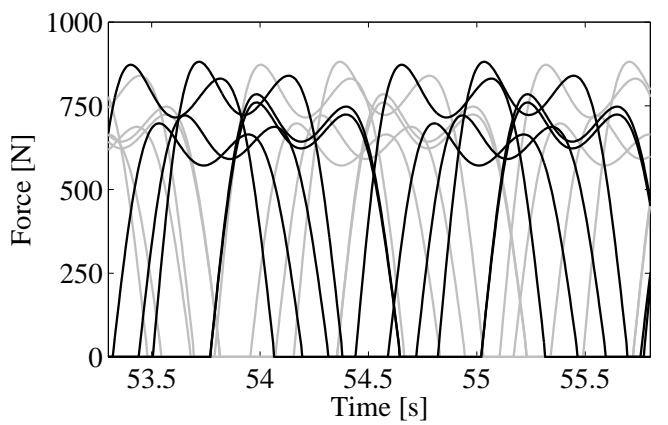

Figure 16: Identified pacing rate for a setup considering synchronised walking $\left(f_{\text {metronome }}=\tilde{f}_{2} / 2=1.49 \mathrm{~Hz}\right)$ : (a) each step of every test-subject indicated by a single vertical line and for a phase characterised by a (b-d) high and a (c-e) low synchronisation rate: (b-c) accelerations measured at the front of the torso for the 6 test-subjects and (d-e) corresponding simulated vertical forces induced by the left (grey) and right (black) foot.

the setup where the subjects were walking two by two, with a targeted pacing rate equal to $1 / 2 \tilde{f}_{2}$. Again, three results are presented: the simulated acceleration (1) due to the perfectly periodic forces, (2) due to the imperfect near-periodic walking forces, and (3) the measured response. This figure also illustrates that the response of the structure is overestimated when the forces are assumed to be perfectly periodic. Accounting for imperfect walking behaviour by applying the identified pacing rate improves the agreement with the measured response significantly although the predicted vibration levels are three times larger. Comparing the results in the frequency domain (figure 18-b) shows that both simulated and measured responses are dominated by the contribution of the second mode $\left(\tilde{f}_{2}\right)$.

An explanation for the remaining discrepancy between measured and simulated structural response is to be found in one or more of the components of the simulation model. The structural modelling errors mainly affect natural frequencies and mode shapes of higher modes, and their impact is negligible as the structural response is dominated by the first or second mode of the 
footbridge. The identified structural damping ratios are however subject to a significant degree of uncertainty [26] and the simulation of the imperfect real walking forces results into some small differences due to the application of the generalised force model. The difference in amplitude between the predicted and measured response in figure 18, is striking and cannot simply result from these remaining uncertainties. It can however be explained by an increased damping. This increase can be due to the presence of the pedestrians [31] but structural damping has also been found to increase with amplitude [34]. The free decay analyses show, however, that the damping characteristics of this structure hardly depend on the vibration amplitudes.

The estimation of the modal damping ratios under human occupation in section 3.1 shows that the presence of the test-subjects leads to a significant increase of the modal damping ratios. Additional numerical simulations are therefore performed with increased modal damping ratios. The best fit between the predicted and measured response is found for $\xi_{1}=2.9 \%$ and $\xi_{2}=0.8 \%$, values that can be justified based on the analysis presented in section 3.1. Figure 19 presents the best fit obtained for the two cases as discussed above. For the case where the step frequency of the test-subjects was chosen to match the first mode of the structure, this increase in modal damping ratio now also results into an good quantitative agreement. For the second mode, the agreement between predicted and measured response has improved but not to the full extent. This may be due to the fact that the added damping resulting from the presence of the pedestrians depends on their distribution on the bridge deck. These results show however that the remaining discrepancy between the simulated and measured response can be mainly attributed to an increase in structural damping. This increase can be explained by the changes in dynamic properties of the combined human-structure system compared to the empty structure.

\section{Conclusions}

The walking behaviour of pedestrians is analysed in situ by $3 \mathrm{D}$ inertial motion tracking. The technique is first tested in laboratory conditions where it was found that it enables to identify the average step frequency and pacing rate of the test-subject. The simulation of the ground reaction forces based on a generalised force model available in literature, characterised by the weight of the test-subject and the identified pacing rate, provides a good approximation of the imperfect real walking forces.

Next, in situ experiments are performed on a footbridge where the walking behaviour of (groups of) pedestrians and the structural response are registered simultaneously. The pacing rate and the rate of synchronisation among the pedestrians are identified from the registered motion of the test-subjects. Numerical simulations are performed using a generalised step-by-step force model, a calibrated numerical model of the structure and the in situ identified damping ratios. The results show that assuming perfectly periodic forces leads to a significant overestimation of the structural response. A much better qualitative agreement between the measured response and the simulations is found when imperfect real walking behaviour is accounted for. The remaining discrepancy between measured and predicted response can be explained by the presence of the pedestrians on the footbridge that leads to a significant increase in structural damping.

\section{Acknowledgements}

This research is funded by the Agency for Innovation by Science and Technology in Flanders (IWT). Their financial support is gratefully acknowledged. 


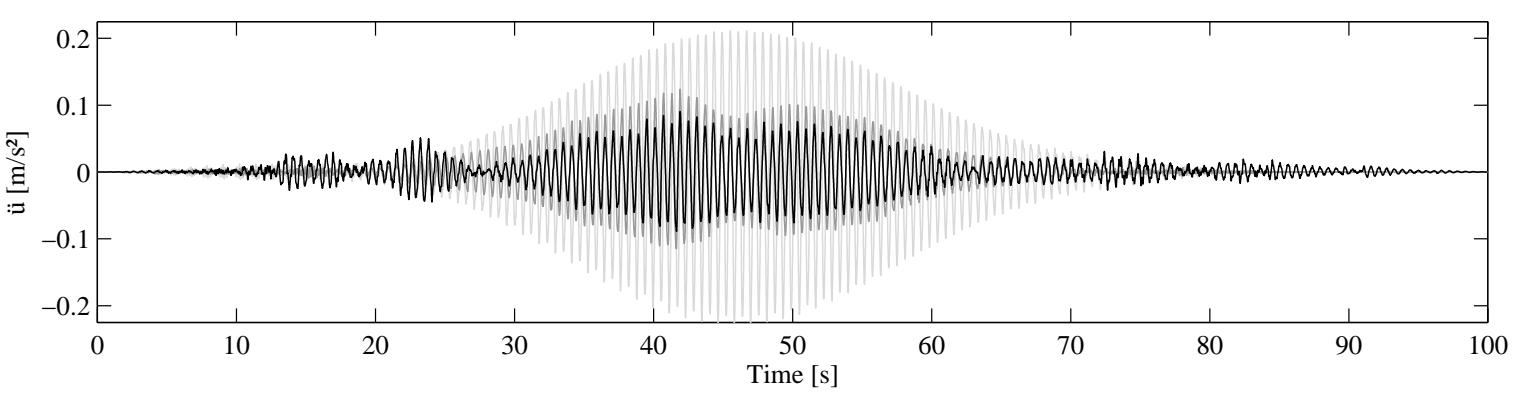

(a)

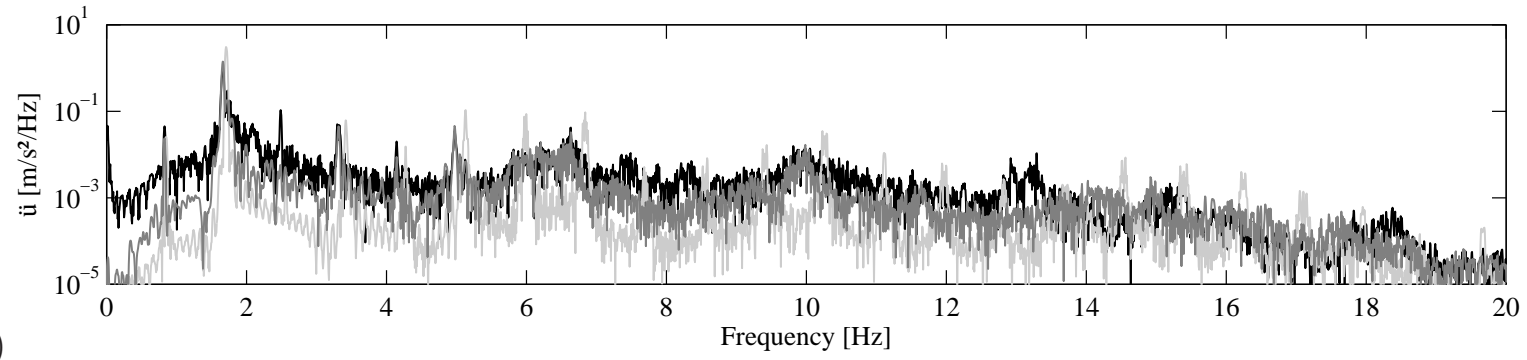

Figure 17: Lateral accelerations levels at midspan for single file walking with a step frequency chosen to match the first mode of the footbridge: time history (a) and linear spectrum (b) of the measured acceleration levels (black), and the simulated acceleration levels accounting for the identified pacing rate of each pedestrian (dark grey) and simulated only applying the identified mean step frequency (light grey). 


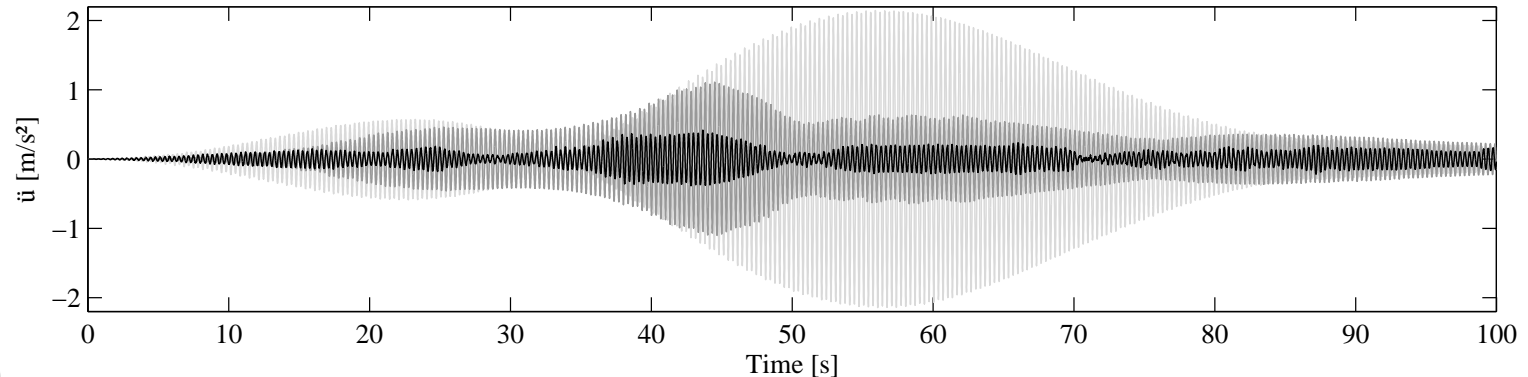

(a)

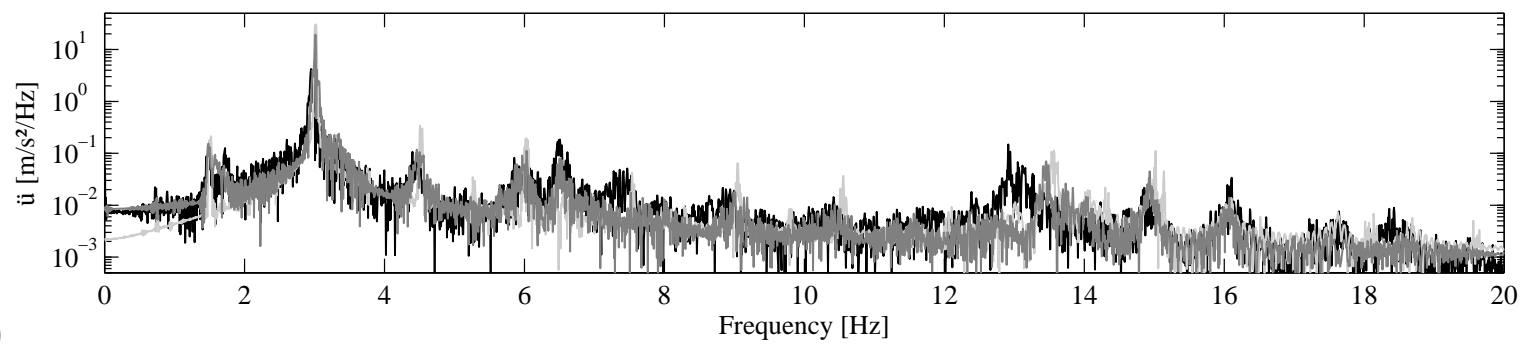

Figure 18: Vertical accelerations levels at midspan for two by two walking with a step frequency chosen to match the second mode of the footbridge: time history (a) and linear spectrum (b) of the measured acceleration levels (black), and the simulated acceleration levels accounting for the identified pacing rate of each pedestrian (dark grey) and simulated only applying the identified mean step frequency (light grey). 


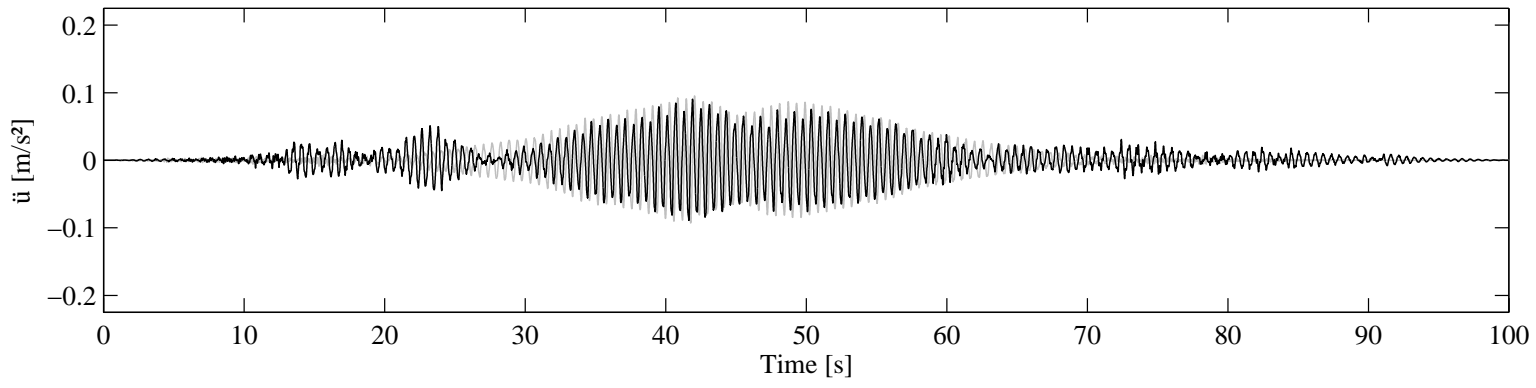

(a)

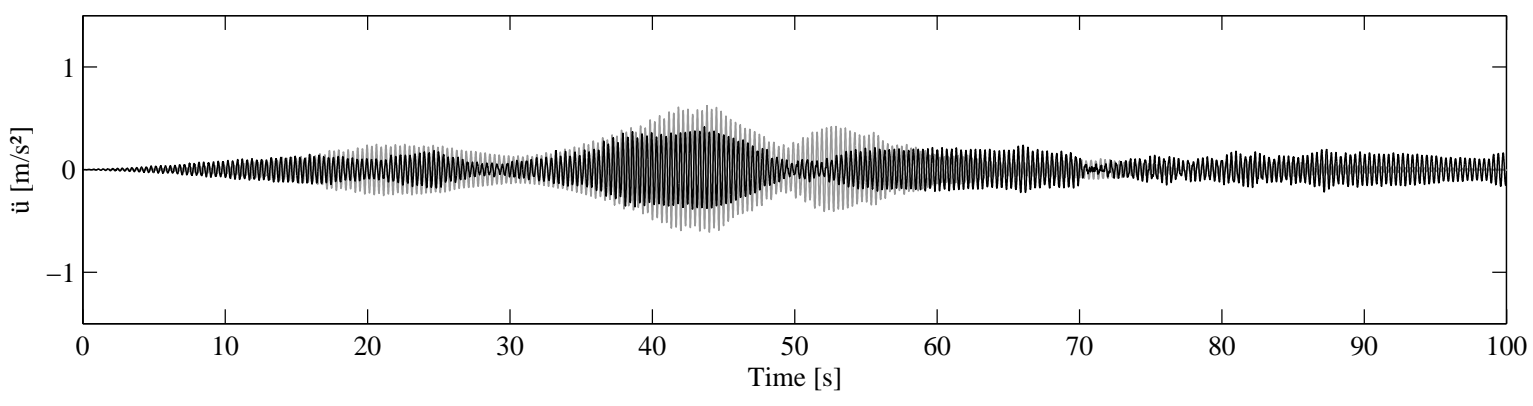

Figure 19: Time series of the lateral (a) and vertical (b) acceleration levels at midspan for single file walking with a step frequency chosen to match the first mode of the footbridge (a) and two by two walking with a step frequency chosen to match the second mode of the footbridge (b): measured (black) and simulated (grey) accounting for the identified pacing rate of each pedestrian and increased modal damping ratios $\left(\xi_{1}=2.9 \%\right.$ and $\left.\xi_{2}=0.8 \%\right)$. 


\section{References}

[1] J. M. W. Brownjohn, P. Fok, M. Roche, and P. Moyo, "Long span steel pedestrian bridge at Singapore Changi Airport - part 1: Prediction of vibration serviceability problems," The Structural Engineer, vol. 82, no. 16, pp. 21-27, 2004.

[2] P. Van den Broeck, K. Van Nimmen, B. Gezels, E. Reynders, and G. De Roeck, "Measurement and simulation of the human-induced vibrations of a footbridgee," in Proceedings of the 8th International Conference on Structural Dynamics EURODYN 2011 (G. De Roeck, G. Degrande, G. Lombaert, and G. Müller, eds.), (Leuven, Belgium), pp. 941-948, July 2011. CD-ROM.

[3] E. Caetano, A. Cunha, F. Magalhães, and C. Moutinho, "Studies for controlling human-induced vibration of the Pedro e Inês footbridge, Portugal. Part 1: Assessment of dynamic behaviour," Engineering Structures, vol. 32, pp. 1069-1081, 2010.

[4] S. Živanović, A. Pavić, and P. Reynolds, "Vibration serviceability of footbridges under human-induced excitation: a literature review," Journal of Sound and Vibration, vol. 279, no. 1-2, pp. 1-74, 2005.

[5] G. Piccardo and F. Tubino, "Simplified procedures for vibration serviceability analysis of footbridges subjected to realistic walking loads," Computers and Structures, vol. 87, pp. 890-903, 2009.

[6] Association Française de Génie Civil, Sétra/AFGC, Sétra: Evaluation du comportement vibratoire des passerelles piétonnes sous l'action des piétons (Assessment of vibrational behaviour of footbridges under pedestrian loading), 2006.

[7] C. Butz, M. Feldmann, C. Heinemeyer, and G. Sedlacek, "SYNPEX: Advanced load models for synchronous pedestrian excitation and optimised design guidelines for steel footbridges," report, Research Fund for Coal and Steel, 2007.

[8] E. T. Ingólfson, C. T. Georgakis, F. Ricciardelli, and J. Jönsson, "Experimental identification of pedestrianinduced lateral forces on footbridges," Journal of Sound and Vibration, vol. 330, pp. 1265-1284, 2011.

[9] V. Racić, A. Pavić, and P. Reynolds, "Experimental identification and analytical modelling of walking forces: a literature review," Journal of Sound and Vibration, vol. 326, pp. 1-49, 2009.

[10] F. Venuti and L. Bruno, "Crowd-structure interaction in lively footbridges under synchronous lateral excitation: a literature review," Physics of Life Reviews, vol. 6, pp. 176-206, 2009.

[11] Vicon Motion Systems, Vicon Motion Systems Product Manuals. Movement \& posture Analysis Laboratory Leuven (MALL) Interdepartmental research laboratory at the Faculty of Kinisiology and Rehabilitation Sciences - KU Leuven.

[12] CODAmotion, Technical dat sheet. Charnwood Dynamics.

[13] V. Racić, J. M. W. Brownjohn, and A. Pavić, "Reproduction and application of human bouncing and jumping forces from visual marker data," Journal of Sound and Vibration, no. 329, pp. 3397-3416, 2010.

[14] S. P. Carroll, J. S. Owen, and M. F. M. Hussein, "Reproduction of lateral ground reaction forces from visual marker data and analysis of balance response while walking on a laterally oscillating deck," Engineering Structures, vol. 49, pp. 1034-1047, 2013.

[15] J. Duysens, I. Jonkers, and S. Verschueren, MALL: Movement 83 posture Analysis Laboratory Leuven. Interdepartmental research laboratory at the Faculty of Kinisiology and Rehabilitation Sciences - KU Leuven.

[16] Xsens Technologies B. V., MTw User Manual, 2010.

[17] Y. Matsumoto and M. J. Griffin, "Dynamic response of the standing human body exposed to vertical vibration: influence of posture and vibration magnitude," Journal of Sound and Vibration, vol. 212, no. 1, pp. 85-107, 1998.

[18] G. Paolini, U. D. Croce, P. O. Riley, F. K. Neuwton, and D. C. Kerrigan, "Testing of a tri-instrumented-treadmill unit for kinetic analysis of locomotion tasks in static and dynamic loading conditions," Medical Engineering and Physics, no. 29, pp. 404-411, 2007.

[19] S. C. White, H. J. Yack, C. A. Tucker, and H. Y. Lin, "Comparison of vertical ground reaction forces during overground and treadmill walking," Medicine and Science in Sports and Exercise, vol. 30, no. 10, pp. 1537-1542, 1998.

[20] Q. Li, J. Fan, J. Nie, Q. Li, and Y. Chen, "Crowd-induced random vibration of footbridge and vibration control using multiple tuned mass dampers," Journal of Sound and Vibration, no. 329, pp. 4068-4092, 2010.

[21] A. Ebrahimpour, A. Hamam, R. L. Sack, and W. N. Patten, "Measuring and Modeling Dynamic Loads imposed by Moving Crowds," Journal of Structural Engineering, no. 122, pp. 1468-1474, 1996.

[22] C. J. Middleton, Dynamic Performance of High Frequency Floors. PhD thesis, University of Sheffield, 2009.

[23] V. Racić and J. M. W. Brownjohn, "Mathematical modelling of random narrow band lateral excitation of footbridges due to pedestrians walking," Computers and Structures, vol. 90-91, pp. 116-130, 2012. 
[24] B. Peeters and G. De Roeck, "Reference-based stochastic subspace identification for output-only modal analysis," Mechanical Systems and Signal Processing, vol. 13, no. 6, pp. 855-878, 1999.

[25] E. Reynders and G. De Roeck, "Reference-based combined deterministic-stochastic subspace identification for experimental and operational modal analysis," Mechanical Systems and Signal Processing, vol. 22, no. 3, pp. 617$637,2008$.

[26] E. Reynders, R. Pintelon, and G. De Roeck, "Uncertainty bounds on modal parameters obtained from Stochastic Subspace Identification," Mechanical Systems and Signal Processing, vol. 22, no. 4, pp. 948-969, 2008.

[27] L. Bruno and F. Venuti, "Crowd-structure interaction in footbridges: Modelling, application to real case-study and sensitivity analysis," Journal of Sound and Vibration, vol. 323, pp. 475-493, 2009.

[28] S. Živanović, I. M. Diaz, and A. Pavić, "Influence of walking and standing crowds on structural dynamic properties," in Proceedings of theIMAC-XXVII, (Florida, USA), February 2009.

[29] E. T. Ingólfsson, C. T. Georgakis, and J. Jönsson, "Pedestrian-induced lateral vibrations of footbridges: A literature review," Engineering Structures, vol. 45, pp. 21-52, 2012.

[30] M. Bocian, J. Macdonald, and J. Burn, "Biomechanically-Inspired Modelling of Pedestrian-Induced Vertical Self-Excited Forces," Journal of Bridge Engineering, vol. 18, pp. 1336-1346, 2013.

[31] J. M. W. Brownjohn, P. Fok, M. Roche, and P. Omenzetter, "Long span steel pedestrian bridge at Singapore Changi Airport - part 2: Crowd loading tests and vibration mitigation measures," The Structural Engineer, vol. 82, no. 16, pp. 28-34, 2004.

[32] F. Magalhães, A. Cunha, E. Caetano, and R. Brincker, "Damping estimation using free decays and ambient vibration tests," Mechanical Systems and Signal Processing, vol. 24, pp. 1274-1290, 2010.

[33] C. Papadimitriou and G. Lombaert, "The effect of prediction error correlation on optimal sensor placement in structural dynamics," Mechanical Systems and Signal Processing, 2011.

[34] S. Živanović, A. Pavić, and P. Reynolds, "Modal testing and FE model tuning of a lively footbridge structure," Engineering Structures, vol. 28, pp. 857-868, 2006. 


\section{List of Tables}

1 Summary of the different walking speeds, the mean step frequency, the number of registered steps and the $95 \%$ confidence interval of the identified onset of each step based on the motion registered at the lower back and near the ankles, for each trial.

2 Identified modal characteristics (mode number $\tilde{N}$, natural frequencies $\tilde{f}$, damping ratios $\tilde{\xi}$ ) of all modes with a natural frequency below $13 \mathrm{~Hz}$ and corresponding mode number $N$ and natural frequencies $f$ predicted by the calibrated numerical model, the calculated Modal Assurance Criterion (MAC) and the relative frequency deviation $\varepsilon \ldots \ldots \ldots \ldots \ldots \ldots \ldots$

3 Identified natural frequency $\tilde{f}_{1}$ and modal damping ratio $\tilde{\xi}_{1}$ of the first combined lateral-torsional mode, provided by the exponential fitting of the corresponding free decay. . . . . . . . . . . . . . . . . . . .

4 Identified natural frequency $\tilde{f}_{2}$ and modal damping ratio $\tilde{\xi}_{2}$ of the first vertical bending mode, provided by the exponential fitting of the corresponding free decay.

\section{List of Figures}

1 Xsens - MTw Development Kit consisting of multiple wireless inertial units (MTw's) and specially designed click-in full body straps (reproduced with permission of Xsens Technologies B.V. [16]). . . . . . . . . . . . . . . . . .

2 Measurement of ground reaction forces: (a) instrumented split-belt treadmill and (b) three force plates (artificially accentuated) integrated in the walkway. . . . . . 3 Configuration setup of the wireless inertial units (MTw's) in laboratory conditions.

4 (a) Ground reaction forces registered by the force plates with a sampling frequency of $1 \mathrm{kHz}$ for the left (-) and right (--) foot respectively, normalised to the weight of the test-subject $G$, for a self-selected walking speed and (b) corresponding linear spectrum of a single step: anteroposterior (black), mediolateral (light grey) and vertical (dark grey) component. . . . . . . . . . . . . . . . . . . . .

5 Ground reaction forces for a walking speed of $5 \mathrm{~km} / \mathrm{h}$ for the left (-) and right (-.) foot respectively, registered by the instrumented split-belt treadmill: (a) original signal with a sampling frequency of $1 \mathrm{kHz}$ and (b) decimated by a factor of ten and low-pass filtered with a cut-off frequency of $12 \mathrm{~Hz}$ : anteroposterior (black), mediolateral (light grey) and vertical (dark grey) component. . . . . . . . . . . .

6 Linear spectrum up to $15 \mathrm{~Hz}$ of (a) the vertical ground reaction forces (sum of left and right foot) registered by the instrumented split-belt treadmill for a walking speed of $5 \mathrm{~km} / \mathrm{h}$ and (b) the corresponding measured vertical acceleration levels at the lower back. . . . . . . . . . . . . . . . . . . . . . .

7 For a walking speed of $5 \mathrm{~km} / \mathrm{h}$ : (a-b) normalised ground reaction forces registered by the instrumented split-belt treadmill, (c-d) normalised measured accelerations at the lower back for the longitudinal (black), lateral (light grey) and vertical (dark gray) component and (e-f) normalised magnitude of the total acceleration vector measured near the left (black) and right (gray) ankle. . . . . . . . . . . . . 
8 For a normal walking speed: (a-b) normalised ground reaction forces registered by the force plates, (c-d) normalised measured accelerations at the lower back for the longitudinal (black), lateral (light grey) and vertical (dark gray) component and (e-f) normalised magnitude of the total acceleration vector measured near the left (black) and right (gray) ankle. . . . . . . . . . . . . . . . . . .

9 The measured vertical ground reaction forces for a walking speed of $5 \mathrm{~km} / \mathrm{h}$ (black) and the corresponding simulated vertical walking forces with step frequency and time in between two successive steps identified based on the tracked motion of the body segments of the test-subject (grey). . . . . . . . . . . . .

10 Linear spectrum of the measured vertical ground reaction forces for a walking speed of $5 \mathrm{~km} / \mathrm{h}$ (black) and the corresponding simulated vertical walking forces according to $\mathrm{Li}$ et al. [20] with mean step frequency $f_{s}$ and pacing rated identified based on the tracked motion of the body segments of the test-subject (grey) . . . . . . . .

11 Eeklo footbridge (a) with cross section (b). . . . . . . . . . . . . .

12 Plan view of the Eeklo footbridge including the location of the roving and reference sensors (dark). . . . . . . . . . . . . . . . . . . . . .

13 Top and side view of the first six identified modes of the Eeklo footbridge: (a) Mode 1 with $\tilde{f}_{1}=1.71 \mathrm{~Hz}$, (b) Mode 2 with $\tilde{f}_{2}=2.99 \mathrm{~Hz}$, (c) Mode 3 with $\tilde{f}_{3}=3.25 \mathrm{~Hz}$, (d) Mode 4 with $\tilde{f}_{4}=3.46 \mathrm{~Hz}$, (e) Mode 5 with $\tilde{f}_{5}=5.77 \mathrm{~Hz}$ and (f) Mode 6 with $\tilde{f}_{6}=5.82 \mathrm{~Hz} \ldots \ldots \ldots \ldots \ldots \ldots \ldots$

14 Measured vertical free vibration response of the footbridge at midspan including the presence of 15 test-subjects (a-c) with straight legs and (d-f) slightly bent legs: (a, d) time series with selected extremes, (b, e) corresponding amplitude spectrum and $(\mathrm{c}, \mathrm{f})$ natural logarithm and best-fit line of peak amplitudes $\left(y_{i}\right)$ with peak points

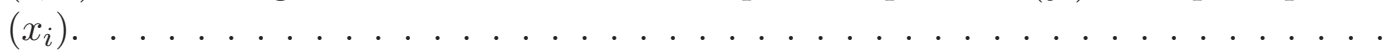

15 Synchronised walking of 6 persons (a) two by two and (b) in a row. . . . . . . . 16 Identified pacing rate for a setup considering synchronised walking $\left(f_{\text {metronome }}=\right.$ $\tilde{f}_{2} / 2=1.49 \mathrm{~Hz}$ ): (a) each step of every test-subject indicated by a single vertical line and for a phase characterised by a (b-d) high and a (c-e) low synchronisation rate: (b-c) accelerations measured at the front of the torso for the 6 test-subjects and (d-e) corresponding simulated vertical forces induced by the left (grey) and right

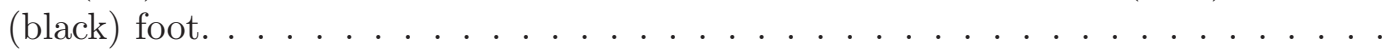

17 Lateral accelerations levels at midspan for single file walking with a step frequency chosen to match the first mode of the footbridge: time history (a) and linear spectrum (b) of the measured acceleration levels (black), and the simulated acceleration levels accounting for the identified pacing rate of each pedestrian (dark grey) and simulated only applying the identified mean step frequency (light grey). . . . . . . .

18 Vertical accelerations levels at midspan for two by two walking with a step frequency chosen to match the second mode of the footbridge: time history (a) and linear spectrum (b) of the measured acceleration levels (black), and the simulated acceleration levels accounting for the identified pacing rate of each pedestrian (dark grey) and simulated only applying the identified mean step frequency (light grey). . . . . . . 
19 Time series of the lateral (a) and vertical (b) acceleration levels at midspan for single file walking with a step frequency chosen to match the first mode of the footbridge (a) and two by two walking with a step frequency chosen to match the second mode of the footbridge (b): measured (black) and simulated (grey) accounting for the identified pacing rate of each pedestrian and increased modal damping ratios

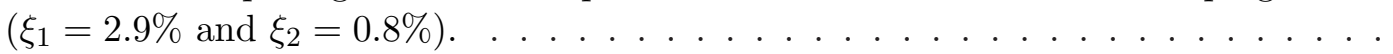

